\title{
STAR-FINITE REPRESENTATIONS OF MEASURE SPACES
}

BY

\author{
ROBERT M. ANDERSON ${ }^{1}$
}

\begin{abstract}
In nonstandard analysis, ${ }^{*}$-finite sets are infinite sets which nonetheless possess the formal properties of finite sets. They permit a synthesis of continuous and discrete theories in many areas of mathematics, including probability theory, functional analysis, and mathematical economics. *-finite models are particularly useful in building new models of economic or probabilistic processes.

It is natural to ask what standard models can be obtained from these *-finite models. In this paper, we show that a rich class of measure spaces, including the Radon spaces, are measure-preserving images of ${ }^{*}$-finite measure spaces, using a construction introduced by Peter A. Loeb [15]. Moreover, we show that a number of measure-theoretic constructs, including integrals and conditional expectations, are naturally expressed in these models. It follows that standard models which can be expressed in terms of these measure spaces and constructs can be obtained from

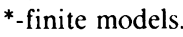

1. Introduction. One of the first goals of nonstandard analysts was to develop a theory of measure and integration based on *-finite sets, replacing set functions by point masses and integrals by sums.

Allen R. Bernstein and Frank Wattenberg [5] constructed Lebesgue measure on $[0,1]$ in the following way. They constructed a *-finite subset $F$ of ${ }^{*}[0,1]$ with the property that, for any Lebesgue measurable $A \subset[0,1],{ }^{\circ}(|F \cap * A| /|F|)$ is the Lebesgue measure of $A$. They also showed that $F$ can be chosen so that ${ }^{\circ}(|F \cap * A| /|F|)$ is a translation-invariant finitely additive measure defined for all subsets of $[0,1]$.

A number of papers have pursued the approach initiated by Bernstein and Wattenberg. For example, Ward Henson [9] extended their procedure to represent

Received by the editors December 30, 1977 and, in revised form, April 3, 1981.

AMS (MOS) subject classifications (1970). Primary 02H20, 26A98, 28A30, 60G45.

Key words and phrases. Radon measures, measure-preserving maps, martingales, uniform integrability, compactifications, nonstandard analysis.

'This paper is based on Chapter III of the author's dissertation, presented for the degree of Doctor of Philosophy in Yale University under the supervision of Professor S. Kakutani. The author was supported by a Canada Council Doctoral Fellowship. The 1977 draft was prepared while the author was a McMaster Fellow at McMaster University. The author is very grateful to Professors Yoav Benyamini, Donald J. Brown, H. Jerome Keisler, Peter A. Loeb, and K. D. Stroyan for a number of helpful discussions, and to the referee for a number of helpful comments. The 1981 version was prepared while the author was a visitor in the Cowles Foundation for Research in Economics at Yale University, on leave from the Departments of Mathematics and Economics at Princeton University. The research was supported in part by a grant from the National Science Foundation. Theorem 3.3 was presented to the Society on January 22, 1976 as part of a paper entitled "Nonstandard Measure Theory in Economics".

(1) 1982 American Mathematical Society $0002-9947 / 81 / 0000-0381 / \$ 06.00$ 
essentially every finitely additive probability measure. Rohit Parikh and Milton Parnes $[19,20]$ showed that the technique could be used to define the conditional probability $P(A \mid B)$ for any pair of subsets of $[0,1]$, retaining translation invariance.

In a series of papers [12-14], Peter A. Loeb showed how to represent a measure space $(X, 6, \mu)$ by an essentially *-finite space. He constructed a *-finite algebra $Q$ such that $\left\{{ }^{*} B: B \in \mathscr{G}\right\} \subset \mathbb{Q} \subset{ }^{*} \mathscr{G}$, and replaced integration in $X$ by summation in $\left({ }^{*} X,\left(t^{\prime},{ }^{*} \mu\right)\right.$.

The emphasis in the above papers is on applications of the transfer principle to relate the properties of $X$ and ${ }^{*} X$. For example, properties of $A \in$ or $f: X \rightarrow R$ are related to properties of ${ }^{*} A$ and ${ }^{*} f$. This use of the transfer principle leads to some difficulties. The basic problem is that countable unions are not preserved under transfer. For example, $\cup_{n=1}^{\infty} A_{n} \neq *\left(\cup_{n=1}^{\infty} A_{n}\right)$. Indeed, a countable union of internal sets is never internal unless it reduces to a finite union.

However, all set theory is preserved under inverse images of functions, and a good part of set theory is preserved under direct images. Measure-preserving maps are a natural way to handle measure-theoretic problems because they preserve the structures important in measure theory.

There are a number of situations in which measure-preserving maps have resolved problems where transfer-based arguments failed. Loeb [16] extracted standard harmonic measure on an ideal boundary and maximal representing measures for positive harmonic functions as distributions of internal measures. The author [1] constructed Wiener measure on $C([0,1])$ as the distribution of an internal measure $\nu$ on ${ }^{*} C([0,1])$; this was possible even though there is a countable $B \subset C([0,1])$ such that $\nu\left({ }^{*} B\right)=1$. It is highly doubtful that either of these constructions could be carried out usefully without the use of measure-preserving maps.

In [1], the author constructed a Brownian motion as the standard part of a ${ }^{*}$-finite random walk $\chi$ defined on a ${ }^{*}$-finite space $\Omega$. He then considered the problem of defining an Itô integral with respect to this Brownian motion. This involves considering functions on $\Omega \times[0,1]$. Using the measure-preserving map st: ${ }^{*}[0,1] \rightarrow$ $[0,1]$, any such function can be lifted to a function on $\Omega \times^{*}[0,1]$. In this setting, the special *-finite properties of $\chi$ can be used to full advantage.

Based on this experience, we believe that measure-preserving maps form a more satisfactory basis for nonstandard measure theory than transfer-based methods. Hence, this paper concentrates on generalizing the specialized techniques used by Loeb $[15,16]$ and the author [1] to permit applications to a wide class of problems.

In [15], Loeb showed how to convert a nonstandard measure space $\left(Y, C^{\prime}, \nu\right)$ to a standard measure space $(Y, L(\mathbb{\psi}), L(\nu))$. The theory of these Loeb spaces was further studied by the author [1]. We shall show that, under suitable hypotheses, the standard part map st: $\left({ }^{*} X, L\left({ }^{*}, h\right), L\left({ }^{*} \mu\right)\right) \rightarrow(X, \mathscr{h}, \mu)$ is measure-preserving. In particular, this is true if $X$ is Radon, confirming a conjecture of Donald J. Brown.

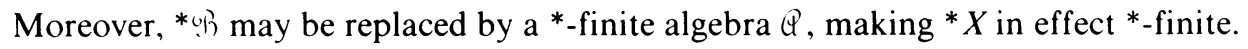

Thus, we shall see that most measure spaces, including all Radon spaces, can be represented by *-finite Loeb spaces. In $§ \S 5-7$ we discuss a number of techniques for representing standard measure-theoretic constructs and manipulating Loeb spaces. 
Among the items considered are integration, measurability, and conditional expectations. These techniques are abstracted from special techniques used by Loeb [15] and the author [1]. In a number of cases, they were developed specifically in response to problems in mathematical economics posed by Brown.

In $\$ 7$, we also show that any martingale can be represented by a *-finite martingale. This representation takes a special form if the underlying probability space is Radon. Douglas N. Hoover and Edwin Perkins [25] and Tom L. Lindstrom [26] have recently developed stronger representations for martingales appropriate to the theory of stochastic integration.

In $\S 8$, we study a special class of internal measures, which we call standardly distributed. These measures have many of the properties of the stars of standard measures. Almost all *-finite samples from a standard distribution are standardly distributed. The original motivation for the study was a problem in core theory in mathematical economics [3], but we anticipate the notion will be useful in other contexts.

In another article [4], Salim Rashid and the author have given a nonstandard characterization of weak convergence.

H. Jerome Keisler has proposed the extensive use of *-finite sets for building models in probability and the social sciences. Our results show that the class of models which can be obtained in this way is at least as rich as the class obtainable through the measure-theoretic constructs we have represented. Applications to mathematical economics are given in Chapter $\mathrm{V}$ of the author's dissertation [2], in [3], and in Rashid [21].

Keisler [11] and Douglas N. Hoover [10] have studied the model theory of *-finite probability spaces, concentrating on properties expressible in certain formal languages. Their results apply to a wider class of measure spaces and give a more systematic characterization of the properties preserved. However, certain properties expressible in terms of our constructions are not expressible in those languages, and are important in applications to economics. Thus, our results complement theirs.

2. Loeb spaces. In this section, we sketch and extend Loeb's construction for converting a nonstandard measure space $(X, \mathcal{Q}, \nu)$ to a standard space. Assume that we are given a structure including the real numbers and a fixed denumerably comprehensive enlargement of this structure. Let $X$ be an internal set in this enlargement, $\mathcal{Q}$ an internal algebra of subsets of $X$, and $\nu: \mathcal{Q} \rightarrow{ }^{*}[0, \infty]$ an internal, additive set function. Then $X, \mathcal{Q}$, and ${ }^{\circ} \nu$ are respectively a set, algebra, and set function in standard set theory. Let $\sigma(Q)$ denote the standard $\sigma$-algebra generated by $\mathcal{Q}$. Using the Carathéodory Extension Theorem, Loeb showed that ${ }^{\circ} \nu$ has a unique extension $L(\nu)$ to $\sigma(Q)$.

In [1], the author considered the measure space $(X, L(\mathbb{Q}), L(\nu))$, which is defined to be the completion of $(X, \sigma(Q), L(\nu))$. It is now convenient to introduce a new $\sigma$-algebra $L^{\prime}(\mathbb{Q})$, satisfying $\sigma(\mathbb{Q}) \subset L^{\prime}(\mathbb{Q}) \subset L(\mathbb{Q})$, so that points not separated by $\mathbb{Q}$ are not separated by $L^{\prime}(\mathbb{Q})$. A special case of this construction was introduced in the definition of $\mathscr{W}_{t}$ in $[1, \S 4]$. Since the proofs are straightforward, they will be omitted; the interested reader may refer to [2, §III.2]. 
DEFINITION 2.1. Let $\sim$ be the equivalence relation on $X$ defined by $x \sim y$ if $x$ and $y$ are not separated by $(\mathcal{Q}$. In other words, $x \sim y$ if and only if, for each $A \in \mathbb{Q}$, $x \in A$ exactly when $y \in A$. Let $[x]$ be the equivalence class of $x$. Let $\mathscr{R}(\Theta)$ be the algebra of unions of equivalence classes. In other words, $\Re(\Theta)=\{B \subset X: B=$ $\left.\cup_{x \in B}[x]\right\}$. Let $L^{\prime}(\mathbb{Q})=L(\mathbb{Q}) \cap \mathcal{P R}(\mathbb{Q})$.

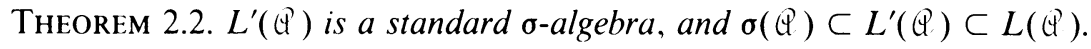

Definition 2.3. Let $X / \sim=\{[x]: x \in X\}, \pi: X \rightarrow X / \sim$ the quotient map $\pi(x)=[x]$.

LeMma 2.4. $B \in \mathbb{M}\left(\left(^{\prime}\right)\right.$ if and only if $\pi^{-1} \pi(B)=B$.

Definition 2.5. Let $Q / \sim=\left\{\pi(A): A \in \mathcal{Q}^{\prime}\right\}$, and let $(\nu / \sim):\left(\mathcal{P} / \sim \rightarrow{ }^{*}[0, \infty]\right.$ be defined by $(\nu / \sim)(\pi(A))=\nu(A)$.

THEOREM 2.6. $\mathrm{C}^{\mathrm{P}} / \sim$ is an internal algebra, and $\nu / \sim$ is an additive set function.

THEOREM 2.7. $\pi:\left(X, L^{\prime}\left(\Theta^{\prime}\right), L(\nu)\right) \rightarrow(X / \sim, L(\Theta / \sim), L(\nu / \sim))$ is a measure isomorphism in the sense that

$$
\begin{array}{cl}
\pi\left(L^{\prime}(\mathbb{Q})\right)=L\left(\Theta^{\prime} / \sim\right), & L^{\prime}\left(\Theta^{\prime}\right)=\pi^{-1}(L(\Theta / \sim)), \\
L(\nu)=L(\nu / \sim) \pi, & L(\nu) \pi^{-1}=L(\nu / \sim) .
\end{array}
$$

REMARK 2.8. All the results in $[1, \S 2]$ remain true with $L^{\prime}\left(\Psi^{\prime}\right)$ substituted for $L\left(\mathcal{A}^{\prime}\right)$, as is readily shown using the isomorphism given by Theorem 2.7. This is of primary interest if $\left(t^{\prime}\right.$ is *-finite. In this case, $X / \sim$ is a *-finite set, and we have the following corollary.

Corollary 2.9. If $\Psi^{\prime}$ is ${ }^{*}$-finite, $\left(X, L^{\prime}(\Theta), L(\nu)\right)$ is measure isomorphic to the Loeb space of $a^{*}$-finite space, namely $\left(X / \sim, L\left(\mathrm{Q}^{\prime} / \sim\right), L(\nu / \sim)\right)$.

3. Radon measures. In this section, we show that a large class of measure spaces are measure-preserving images of *-finite Loeb spaces. We begin by recalling some definitions and results of Abraham Robinson [22, pp. 90-94].

Let $X$ be a Hausdorff space, with $T$ the collection of open sets. For $x \in X$, the monad of $x$ is defined to be

$$
m(x)=\bigcap_{x \in N \in T} * N
$$

If $y \in{ }^{*} X$ and $y \in m(x)$ for some $x \in X$, we write $x={ }^{\circ} y=\operatorname{st}(y)$ and say that $x$ is the standard part of $y$. ${ }^{\circ} y$ is unique. Any $y$ having a standard part is called near-standard; the collection of near-standard points in ${ }^{*} X$ is denoted $n s\left({ }^{*} X\right)$. A subset $A$ of $X$ is compact if and only if ${ }^{\circ} a \in A$ for all $a \in *^{*} A$. $A$ is closed if and only if ${ }^{\circ} a \in A$ for all $a \in *^{*} A \cap \operatorname{ns}\left({ }^{*} X\right)$. $A$ is open if and only if $m(a) \subset{ }^{*} A$ for all $a \in A$.

Next we need some measure-theoretic notions. Since terminology varies in the literature, we include definitions. Suppose that $(X, 6, \mu)$ and $\left(X^{\prime}, 96^{\prime}, \mu^{\prime}\right)$ are measure spaces. A map $T: X^{\prime} \rightarrow X$ is called measure-preserving if $T$ is measurable and $\mu^{\prime}\left(T^{-1}(B)\right)=\mu(B)$ for all $B \in S^{\prime}$. 
A probability space $(X, \Re, \mu)$ is called Radon if

(i) $X$ is a Hausdorff space, $\mathscr{B}$ the Borel $\sigma$-algebra (i.e., the $\sigma$-algebra generated by $\mathcal{T}$, the class of open sets),

(ii) for all $B \in \mathscr{B}$,

$$
\mu(B)=\sup \{\mu(C): C \subset B, C \text { compact }\}=\inf \{\mu(T): T \supset B, T \in \mathcal{T}\} .
$$

Most common probability spaces are Radon. For example, it is shown in Billingsley [6] that any probability measure on the Borel $\sigma$-algebra of a complete separable metric space is Radon. A probability measure $\mu$ is said to be tight if $\sup \{\mu(C): C$ compact $\}=1$. Any tight probability on the Borel $\sigma$-algebra of a metric space is Radon.

Definition 3.1. Let $Q$ be an internal algebra of subsets of ${ }^{*} X$. Recall the equivalence relation $\sim$ of Definition 2.1. $Q$ is said to be $S$-separating if $[x] \subset m\left({ }^{\circ} x\right)$ for all $x \in \mathrm{ns}\left({ }^{*} X\right)$.

REMARK 3.2. It is easily seen that $Q$ is $S$-separating if and only if $\operatorname{st}^{-1}(A) \in \mathscr{N}(\mathbb{Q})$ for all $A \subset X$. If $X$ is metric, $Q$ is $S$-separating if and only if the diameter of $[x]$ is infinitesimal whenever $x \in \mathrm{ns}\left({ }^{*} X\right)$.

The following theorem was conjectured by Donald J. Brown.

TheOREM 3.3. Let $(X, \Re, \mu)$ be Radon, with completion $(X, \mathcal{C}, \mu)$. Let $\mathbb{Q}$ be any internal subalgebra of $* \mathscr{B}$ such that, for any $T \in \mathcal{T}$, either $* T \in \mathbb{Q}$ or $\mathrm{st}^{-1}(T) \in L(\mathbb{Q})$. Then $L\left({ }^{*} \mu\right)\left(\mathrm{ns}\left({ }^{*} X\right)\right)=1$, and st: $\left({ }^{*} X, L(\mathbb{Q}), L\left({ }^{*} \mu\right)\right) \rightarrow(X, \mathcal{C}, \mu)$ is measure-preserving. If, in addition, $\mathbb{Q}$ is $S$-separating, we may replace $L(\Theta)$ by $L^{\prime}(\mathbb{Q})$.

Proof. Suppose $B \in \mathcal{C}$. We may find sequences $C_{n}$ of compact sets and $T_{n}$ of open sets such that $C_{1} \subset C_{2} \subset \cdots \subset B \subset \cdots \subset T_{2} \subset T_{1}$ and such that $\mu\left(T_{n}-C_{n}\right)$ $\rightarrow 0$.

Since $C_{n}$ is compact and $T_{n}$ is open, ${ }^{*} C_{n} \subset \mathrm{st}^{-1}\left(C_{n}\right) \subset \mathrm{st}^{-1}(B) \subset \mathrm{st}^{-1}\left(T_{n}\right) \subset{ }^{*} T_{n}$. ${ }^{*} \mu\left({ }^{*} T_{n}-{ }^{*} C_{n}\right)=\mu\left(T_{n}-C_{n}\right) \rightarrow 0$. Moreover, either ${ }^{*} C_{n} \in \mathbb{Q} \subset L(\mathbb{Q})$ or $\operatorname{st}^{-1}\left(C_{n}\right) \in$ $L(Q)$ and $L\left({ }^{*} \mu\right)\left(\mathrm{st}^{-1}\left(C_{n}\right)\right) \geqslant{ }^{\circ}\left({ }^{*} \mu\left({ }^{*} C_{n}\right)\right)=\mu\left(C_{n}\right)$; note that ${ }^{*} \mu\left({ }^{*} C_{n}\right)$ is defined even if ${ }^{*} C_{n} \notin Q$, since $\mu$ is a Borel measure and $C_{n}$ is compact, hence closed. Either ${ }^{*} T_{n} \in \mathbb{Q} \subset L(\mathbb{Q})$ or $\mathrm{st}^{-1}\left(T_{n}\right) \in L(\mathbb{Q})$ and $L\left({ }^{*} \mu\right)\left(\mathrm{st}^{-1}\left(T_{n}\right)\right) \leqslant{ }^{\circ}\left({ }^{*} \mu\left({ }^{*} T_{n}\right)\right)=\mu\left(T_{n}\right)$. Hence $\mathrm{st}^{-1}(B) \in L(Q)$ and $L\left({ }^{*} \mu\right)\left(\mathrm{st}^{-1}(B)\right)=\mu(B)$. This shows that $L\left({ }^{*} \mu\right)\left(\mathrm{ns}\left({ }^{*} X\right)\right)$ $=1$ and that $s t$ is measure-preserving.

Suppose that $Q$ is $S$-separating. Then $\operatorname{st}^{-1}(B) \in \mathscr{N}(\mathcal{Q})$. Therefore $\operatorname{st}^{-1}(B) \in$ $\mathfrak{N}(\mathbb{Q}) \cap L(\mathbb{Q})=L^{\prime}(\mathbb{Q})$, so the theorem is true with $L(\mathbb{Q})$ replaced by $L^{\prime}(\mathbb{Q})$.

Corollary 3.4. Let $(X, \Re, \mu)$ be Radon with completion $(X, \mathcal{C}, \mu)$. Then there is a ${ }^{*}$-finite probability space $(Y, \Theta, \nu)$ and a measure-preserving map $S:(Y, L(\Theta), L(\nu))$ $\rightarrow(X, \mathcal{e}, \mu)$.

Proof. Loeb [13] observed there is a *finite algebra $Q_{1}$ such that $\left\{{ }^{*} C: C \in \mathcal{C}\right\} \subset$ $Q_{1} \subset * C$; to see this, note that for any finite collection $\left\{C_{1}, \ldots, C_{n}\right\} \subset \mathcal{C}$, there is a

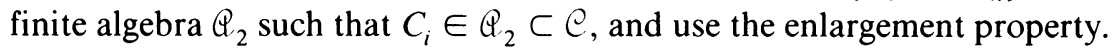

We now show that $Q_{1}$ is $S$-separating. Suppose $x \in \mathrm{ns}\left({ }^{*} X\right)$. Let $T$ be an open neighborhood of ${ }^{\circ} x$. Since $T$ is open, $m\left({ }^{\circ} x\right) \subset{ }^{*} T$. Therefore $x \in{ }^{*} T$; since ${ }^{*} T \in \mathbb{Q}_{1}$, $[x] \subset{ }^{*} T$. Therefore $[x] \subset \cap_{0_{x \in T \in T}}{ }^{*} T=m\left({ }^{\circ} x\right)$. Thus, $\mathbb{Q}_{1}$ is $S$-separating. 
Therefore, st: $\left({ }^{*} X, L^{\prime}\left(\bigotimes_{1}\right), L\left({ }^{*} \mu\right)\right) \rightarrow(X, \mathcal{C}, \mu)$ is measure-preserving by Theorem 3.3. Let $Y={ }^{*} X / \sim, \mathbb{Q}=\mathbb{Q}_{1} / \sim, \nu={ }^{*} \mu / \sim$, and $\pi$ the quotient map $\pi:{ }^{*} X \rightarrow Y$. Let $S=$ st $\circ \pi^{-1}: Y \rightarrow X$. One easily verifies that $S$ is well defined except on the $L(\nu)$-null set $\pi\left({ }^{*} X-\mathrm{ns}\left({ }^{*} X\right)\right)$. Then since st is measure-preserving and $Q$ is a measure isomorphism by Theorem $2.7, S$ is measure-preserving.

Corollary 3.5 [1, Theorem 14]. Let $([0,1]$, e, $\mu)$ be the Lebesgue measure space, and pick $\omega \in{ }^{*} N-N$. Let $\mathbb{Q}$ be the ${ }^{*}$-algebra of subsets of ${ }^{*}[0,1]$ generated by the partition $\{[0,1 / \omega), \ldots,[(\omega-1) / \omega, 1]\}$. Then st: $\left({ }^{*}[0,1], L^{\prime}(\mathbb{Q}), L\left({ }^{*} \mu\right)\right) \rightarrow$ $([0,1], \mathcal{C}, \mu)$ is measure-preserving.

Proof. Since each equivalence class mod $\sim$ has diameter $1 / \omega, Q$ is $S$-separating. $Q \subset * \circlearrowright . \mu$ is Radon. If $a<b \in[0,1]$,

$$
\mathrm{st}^{-1}((a, b))=\bigcup_{n=1}^{\infty}\left[a_{n} / \omega, b_{n} / \omega\right)
$$

where $a_{n}=[(a+1 / n) \omega]$ and $b_{n}=[(b-1 / n) \omega]$, where $[\cdot]$ denotes the greatest integer function. Since $\left[a_{n} / \omega, b_{n} / \omega\right) \in \mathbb{Q}, \mathrm{st}^{-1}((a, b)) \in \sigma(\mathbb{Q})$. Since any open set $T$ is a countable union of open intervals, $\mathrm{st}^{-1}(T) \in \sigma(\mathbb{Q})$. Therefore, st is measure-preserving by Theorem 3.3.

THEOREM 3.6. Let $(X, \Re, \mu)$ be Radon with completion $(X, \bigodot, \mu)$. Let $\Theta$ be any internal algebra containing $\left\{{ }^{*} T: T \in \mathcal{T}\right\}$. Then, for any $B \in \mathcal{C}, \mathrm{st}^{-1}(B) \triangle{ }^{*} B \in L(\mathbb{Q})$ and $L\left({ }^{*} \mu\right)\left(\mathrm{st}^{-1}(B) \triangle{ }^{*} B\right)=0$.

Proof. Let $T_{n}, C_{n}$ be as in the proof of Theorem 3.3. Then ${ }^{*} C_{n} \subset \mathrm{st}^{-1}(B) \subset{ }^{*} T_{n}$ and ${ }^{*} C_{n} \subset{ }^{*} B \subset{ }^{*} T_{n}$. Thus

$$
\mathrm{st}^{-1}(B) \triangle{ }^{*} B \subset{ }^{*} T_{n}-{ }^{*} C_{n} \text {, and } \quad{ }^{*} \mu\left({ }^{*} T_{n}-{ }^{*} C_{n}\right)=\mu\left(T_{n}-C_{n}\right) \rightarrow 0 .
$$

Since $L(\mathscr{Q})$ is complete, $\mathrm{st}^{-1}(B) \triangle^{*} B \in L(\mathscr{Q})$ and $L\left({ }^{*} \mu\right)\left(\mathrm{st}^{-1}(B) \triangle^{*} B\right)=0$.

The following is an analogue of Lusin's theorem and can be used in situations in which Lusin's theorem is used in standard treatments. A nonstandard proof of Lusin's theorem itself is given in Loeb [14]. I am grateful to the referee for pointing out the converse part of the theorem.

TheOrem 3.7. Let $(X, \Re, \mu)$ be Radon with completion $(X, \mathcal{e}, \mu)$, and suppose $f$ : $X \rightarrow Y$.

(i) If $Y$ is a Hausdorff space with a countable base of open sets and $f$ is -measurable, then ${ }^{\circ}\left({ }^{*} f(x)\right)=f\left({ }^{\circ} x\right)$ for $L\left({ }^{*} \mu\right)$-almost all $x$. In particular, if $\mathcal{Q}$ is any internal algebra containing $\left\{{ }^{*} T: T \in \mathcal{T}\right\}$, st $\circ{ }^{*} f$ is $L(\mathbb{Q})$-measurable, and st $\circ{ }^{*} f=f \circ$ st except on a null set in $L(Q)$.

(ii) Conversely, if $X$ is uniform, $Y$ is metric, and ${ }^{\circ}\left({ }^{*} f(x)\right)=f\left({ }^{\circ} x\right)$ for $L\left({ }^{*} \mu\right)$-almost all $x$, then $f$ is E-measurable.

Proof. (i) Let $\left\{U_{n}\right\}_{n \in N}$ be a countable base for $Y$, with $U_{1}=Y$. Note that

$$
L\left({ }^{*} \mu\right)\left({ }^{*} f^{-1}\left({ }^{*} U_{n}\right) \triangle(f \circ \mathrm{st})^{-1}\left(U_{n}\right)\right)=L\left({ }^{*} \mu\right)\left({ }^{*}\left(f^{-1}\left(U_{n}\right)\right) \triangle \mathrm{st}^{-1}\left(f^{-1}\left(U_{n}\right)\right)\right)=0
$$


by Theorem 3.6. Let

$$
X^{\prime}=\bigcup_{n=1}^{\infty}\left(* f^{-1}\left(* U_{n}\right) \triangle(f \circ \mathrm{st})^{-1}\left(U_{n}\right)\right) .
$$

Thus $L\left({ }^{*} \mu\right)\left(X^{\prime}\right)=0$.

$$
\begin{aligned}
& \text { If } x \notin X^{\prime}, x \in \bigcap_{f\left({ }^{\circ} x\right) \in U_{n}}{ }^{*} f^{-1}\left({ }^{*} U_{n}\right) \text {, so } \\
& { }^{*} f(x) \in \bigcap_{f\left({ }^{\circ} x\right) \in U_{n}}{ }^{*} U_{n}=m\left(f\left({ }^{\circ} x\right)\right) .
\end{aligned}
$$

Therefore ${ }^{\circ}\left({ }^{*} f(x)\right)=f\left({ }^{\circ} x\right)$ for all $x \notin X^{\prime}$. st $\circ{ }^{*} f$ is $L(\mathbb{Q})$-measurable because $f \circ$ st is.

(ii) Let $d$ be the metric on $Y$, and $\mathcal{Q}$ the uniformity on $X$. Fix $n \in N$. There exists $B_{n} \in{ }^{*} \bigodot$ with ${ }^{*} \mu\left({ }^{*} X-B_{n}\right)<1 / 2^{n}$ and $B_{n} \subset\left\{x:{ }^{\circ}\left({ }^{*} f(x)\right)=f\left({ }^{\circ} x\right)\right\}$. If $x, y \in B_{n}$ and $x \simeq y$, then ${ }^{*} f(x) \simeq{ }^{*} f(y)$. Thus, there exists $U_{n} \in * \mathscr{Q}$ such that if $(x, y) \in U_{n}$, $x, y \in B_{n}$, then $d\left({ }^{*} f(x),{ }^{*} f(y)\right)<1 / n$. By transfer, there exists $B_{n} \in \mathcal{C}$ and $U_{n} \in \mathcal{Q}$ with $\mu\left(X-B_{n}\right)<1 / 2^{n}$ such that, if $(x, y) \in U_{n}$ and $x, y \in B_{n}$, then $d(f(x), f(y))$ $<1 / n . \mu\left(X-\bigcap_{n>m} B_{n}\right)<1 / 2^{m} . f$ is continuous on $\bigcap_{n>m} B_{n}$, since $(x, y) \in U_{n}$ implies $d(f(x), f(y))<1 / n$ for $n>m$. Define $f_{m}(x)$ to be $f(x)$ if $x \in \bigcap_{n>m} B_{n}$, and 0 otherwise. Then $f_{m}$ is measurable, and $f_{m} \rightarrow f$ on $\cup_{m} \cap_{n>m} B_{n}$, a set of full measure. Therefore $f$ is $\Theta$-measurable.

The previous results show that Loeb algebras are big enough to represent standard Radon probabilities. The next two results show that Loeb algebras are not too big. The first theorem of this type was proved by Ward Henson. He showed that, if $Q$ is an internal algebra of subsets of a compact Hausdorff space $X$ and $B \in \sigma(Q)$, then $\operatorname{st}(B)$ is in the Souslin closure of the closed subsets of $X$. The following theorem is an easy corollary of that result. Edward Fisher later constructed a direct proof of the theorem in the case that $X$ is the Lebesgue space. The proof we give is essentially Fisher's, in a more general setting.

TheOREM 3.8. Let $(X, \Re, \mu)$ be Radon with completion $(X, \mathcal{C}, \mu)$. Suppose ${ }^{*} X$ is taken in a $\kappa$-saturated enlargement where every $x \in X$ has a neighborhood base of cardinality $<\kappa$. Then $\left\{B \subset X: \mathrm{st}^{-1}(B) \in L(* \mathcal{C})\right\}=\bigodot$.

Proof. Theorem 3.3 shows that $\left\{B \subset X:\right.$ st $\left.^{-1}(B) \in L(* \mathcal{C})\right\} \supset \mathcal{C}$. We now proceed to show the reverse inclusion.

Suppose $B \subset X$ and $\mathrm{st}^{-1}(B) \in L\left({ }^{*} \mathcal{C}\right)$. Fix $\varepsilon \in R_{+}$. There exists $A \in{ }^{*} \circlearrowright$ such that $A \subset \mathrm{st}^{-1}(B)$ and ${ }^{*} \mu(A)>L\left({ }^{*} \mu\right)\left(\mathrm{st}^{-1}(B)\right)-\varepsilon$. Let $C=\operatorname{st}(A) . C$ is closed by $[17$, 3.4.2].

Since $C$ is closed, $C \in \mathcal{C}$. Therefore, by Theorem 3.3, $\mathrm{st}^{-1}(C) \in L\left({ }^{*} \mathcal{C}\right)$ and $L\left({ }^{*} \mu\right)\left(\mathrm{st}^{-1}(C)\right)=\mu(C)$. Since $\mathrm{st}^{-1}(C)=\mathrm{st}^{-1}(\operatorname{st}(A)) \supset A$,

$$
\mu(C)=L\left({ }^{*} \mu\right)\left(\mathrm{st}^{-1}(C)\right) \geqslant{ }^{\circ}\left({ }^{*} \mu(A)\right) \geqslant L\left(*^{*} \mu\right)\left(\mathrm{st}^{-1}(B)\right)-\varepsilon .
$$

Finally, $\left.C=\operatorname{st}(A) \subset \operatorname{st}^{-1}(B)\right)=B$.

We have shown that there exists $C$ closed, $C \subset B, \mu(C) \geqslant L\left({ }^{*} \mu\right)\left(\operatorname{st}^{-1}(B)\right)-\varepsilon$. Now note that

$$
\mathrm{st}^{-1}(X-B)=\mathrm{ns}(* X)-\mathrm{st}^{-1}(B) \in L(* \bigodot)
$$


hence we can apply the same argument to $X-B$ to find $C^{\prime}$ closed, $C^{\prime} \subset X-B$, $\mu\left(C^{\prime}\right) \geqslant L\left({ }^{*} \mu\right)\left(\mathrm{st}^{-1}(X-B)\right)-\varepsilon$. Therefore $X-C^{\prime}$ is open, $X-C^{\prime} \supset B$, and

$$
\mu\left(X-C^{\prime}\right) \leqslant 1-L\left({ }^{*} \mu\right)\left(\mathrm{st}^{-1}(X-B)\right)+\varepsilon=L\left({ }^{*} \mu\right)\left(\mathrm{st}^{-1}(B)\right)+\varepsilon .
$$

This shows that $B$ is in the completion of $\mathscr{B}$ with respect to $\mu$, i.e. that $B \in \mathcal{C}$.

Definition 3.9. Let $S=\left\{\mathrm{st}^{-1}(D): D \subset X\right\}$, and $\delta(\mathcal{Q})=\delta \cap L(Q)$. $S(\mathbb{Q})$ will be called the standard Loeb algebra determined by $Q$.

Note that $\delta(\mathbb{Q})$ is a $\sigma$-algebra of subsets of $n s\left({ }^{*} X\right)$. Note also that, if $\Theta$ is $S$-separating, $S(\mathbb{Q})=\delta \cap L^{\prime}(\mathbb{Q})$.

TheOrem 3.10. Suppose $(X, 93, \mu)$ is Radon with completion $(X, \mathcal{C}, \mu)$, and that ${ }^{*} X$ is taken in a $\kappa$-saturated enlargement where every $x \in X$ has a neighborhood base of cardinality $<\kappa$. Then if $\Theta$ satisfies the assumptions in Theorem 3.3, st: $\left({ }^{*} X, S(\mathbb{Q}), L\left({ }^{*} \mu\right)\right) \rightarrow(X, \mathcal{C}, \mu)$ is a measure isomorphism. There is a ${ }^{*}$-finite probability space $\left(Y, \mathcal{Q}_{1}, \nu\right)$ and a measure isomorphism $\left.S:\left(Y, S_{\left(\Theta_{1}\right.}\right), L(\nu)\right) \rightarrow(X, \mathcal{e}, \mu)$.

Proof. Theorems 3.3, 3.4, and 3.8.

TheOREM 3.11. Let $(X, 9, \mu)$ be Radon with completion $(X, \mathcal{C}, \mu)$. Let ${ }^{*} X$ be taken in a $\kappa$-saturated enlargement, where every $x \in X$ has a neighborhood base of cardinality $<\kappa$. Let $(Y, \mathcal{D})$ be any measurable space. If $g:{ }^{*} X \rightarrow Y$ is $L\left({ }^{*}\right)$-measurable, and $g(x)=g\left({ }^{\circ} x\right)$ for $L\left({ }^{*} \mathcal{C}\right)$-almost all $x$, then $f=\left.g\right|_{X}$ is -measurable and has the same distribution as $g$. Moreover, if $\mathscr{D}$ is the Borel algebra of a Hausdorff topology with a countable base on $Y$, then ${ }^{\circ}\left({ }^{*} f(x)\right)=g(x)$ for $L\left({ }^{*} \mu\right)$-almost all $x$.

Proof. Let $g^{\prime}: \operatorname{ns}\left({ }^{*} X\right) \rightarrow Y$ be defined by $g^{\prime}(x)=g\left({ }^{\circ} x\right)$. Then $g^{\prime}(x)=g(x)$ for $L\left(^{*} \mu\right)$-almost all $x$, so $g^{\prime}$ is $L\left({ }^{*} \mathcal{C}\right)$-measurable and has the same distributions as $g$. But $g^{\prime}$ is $\Im$-measurable, so $g^{\prime}$ is $\Im(* \circlearrowright)$-measurable.

$f=\left.g\right|_{X}=\left.g^{\prime}\right|_{X}=g^{\prime} \circ \mathrm{st}^{-1}$. Since st: $\left({ }^{*} X, \delta\left({ }^{*}\right), L\left({ }^{*} \mu\right)\right) \rightarrow(X, \circlearrowright, \mu)$ is a measure

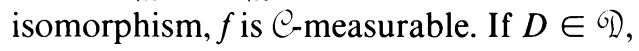

$$
\mu\left(f^{-1}(D)\right)=L\left({ }^{*} \mu\right)\left(\mathrm{st}^{-1}\left(f^{-1}(D)\right)\right)=L\left(*^{*} \mu\right)\left(g^{\prime-1}(D)\right)=L\left(*^{*} \mu\right)\left(g^{-1}(D)\right)
$$

so $g$ and $f$ have the same distribution.

If $\mathscr{D}$ is the Borel algebra of a topology with a countable base, then by Theorem $3.7,{ }^{\circ}\left({ }^{*} f(x)\right)=f\left({ }^{\circ} x\right)$ for $L\left({ }^{*} \mu\right)$-almost all $x$. Therefore ${ }^{\circ}\left({ }^{*} f(x)\right)=f\left({ }^{\circ} x\right)=g\left({ }^{\circ} x\right)=$ $g(x)$ for $L\left({ }^{*} \mu\right)$-almost all $x$.

We close with a note on $\sigma$-finite measure spaces. It is easy to see that no nonfinite Loeb space is $\sigma$-finite. However, if $(X, \mathscr{B}, \mu)$ is a countable union of Radon spaces, we can construct representations on each Radon piece, then glue them together. Alternatively, we can extract a $\sigma$-finite subset of $\left({ }^{*} X, L\left({ }^{*} \mathcal{C}\right), L\left({ }^{*} \mu\right)\right)$. The details are left to the reader.

4. Non-Radon measures. The representations of $\$ 3$ are no longer possible if $(X, \Re, \mu)$ is not Radon. For example, let $c$ be the first uncountable ordinai, $X=\{1, \ldots, c\}, \sigma$ the order topology on $X, 93$ the Borel $\sigma$-algebra. $X$ is a compact 
Hausdorff space. It is well known [18, Problem 17, p. 58] that there is a probability $\mu$ on $\mathscr{B}$ such that for $A \in \mathscr{B}$,

$$
\mu(A)= \begin{cases}1 & \text { if } A \text { contains a closed cofinal subset of } X-\{c\}, \\ 0 & \text { otherwise. }\end{cases}
$$

It is not hard to see that $L\left({ }^{*} \mu\right)\left(\right.$ st $\left.^{-1}(\{c\})\right)=1$, while $\mu(\{c\})=0$.

However, if we restrict $\mu$ to the Baire sets, one easily sees that the standard part map is measure-preserving. This observation provides the point of departure for this section.

The basic approach will be to embed $(X, \mathscr{B}, \mu)$ in a compact Hausdorff space $\hat{X}$; we induce a Baire measure $\hat{\mu}$ on $\hat{X}$ and show that this induced measure is represented in a natural way by a Loeb space on ${ }^{*} X$.

Definition 4.1. Suppose $(X, \mathcal{T})$ is a Hausdorff space. Let $C(X)$ denote the space of all bounded continuous real-valued functions on $X$. The Baire algebra on $X$ is the smallest $\sigma$-algebra such that every $f \in C(X)$ is measurable.

We shall first study compactifications. Our result is an easy extension of results in the nonstandard literature. Since no new ideas are involved in the proof, we omit the details and refer the interested reader to $[18, \S \S 7,9 ; 17,3.15 .4 ; 13$, p. 77 ; and 24 , 8.4.34, 9.2.5].

DEFINITION 4.2. Let $X$ be any set, $\mathscr{F}$ any collection of bounded real-valued functions on $X$. Let ${ }^{*} X$ be the extension of $X$ in a $\kappa$-saturated enlargement, where $\max (|\mathscr{F}|,|N|)<\kappa$. Define an equivalence relation $\propto$ on ${ }^{*} X$ by $x \propto y \Leftrightarrow{ }^{\circ *} f(x)=$ ${ }^{\circ} * f(y)$ for all $f \in \mathcal{F}$. Let $\hat{X}=* X / \propto$. Let $\hat{f}: \hat{X} \rightarrow R$ be defined by $\hat{f}(\hat{x})={ }^{\circ *} f(x)$. Set $\hat{\mathscr{F}}=\{\hat{f}: f \in \mathscr{F}\}$. Let $\mathscr{T}$ be the topology on $\hat{X}$ generated by the subbase $\{\{\hat{x} \in \hat{X}$ : $\hat{f}(\hat{x}) \in T\}: \hat{f} \in \hat{\mathscr{F}}, T \subset R, T$ open $\}$. Let $I: X \rightarrow \hat{X}$ be defined by $I(x)=\widehat{*} x$. Let $\Pi$ : ${ }^{*} X \rightarrow \hat{X}$ be the quotient map.

THEOREM 4.3. (i) $I(X)$ is a dense subset of $(\hat{X}, \hat{\mathcal{J}})$, which is a compact Hausdorff space. If $f \in \mathcal{F}, \hat{f}$ is the unique continuous extension of $f$ to $\hat{X}$.

(ii) If $\mathscr{F}$ separates points in $X, I$ is an injection.

(iii) If $\mathscr{F}$ is an algebra containing $1, \hat{\mathscr{F}}$ is dense in $C(\hat{X})$.

THEOREM 4.4. Suppose $\mathscr{F}$ is an algebra of bounded functions containing 1. Let $\Phi$ be a positive linear functional on $\mathscr{F}$ with $\Phi(1)=1$. Then there is a unique Radon probability $\hat{\mu}$ such that, for all $f \in \mathscr{F}, \Phi(f)=\int_{\hat{X}} \hat{f} d \hat{\mu}$.

Proof. Define $\hat{\Phi}(\hat{f})=\Phi(f)$ for $f \in \mathcal{F}$. Since $\hat{\Phi}$ is positive and $\hat{\Phi}(\hat{1})=1, \hat{\Phi}$ is bounded. Since $\hat{\mathscr{F}}$ is dense in $C(\hat{X})$, $\hat{\Phi}$ extends to a unique bounded functional $\hat{\Phi}$ on $C(\hat{X})$.

$\hat{X}$ is compact Hausdorff. Hence [7], there is a unique Borel measure $\hat{\mu}$ such that $\hat{\Phi}(g)=\int_{\hat{X}} g d \hat{\mu}$ for all $g \in C(\hat{X})$, and such that $\hat{\mu}$ satisfies conditions (i) and (ii) in the definition of Radon; since $\hat{\mu}$ is also obviously a probability, it is Radon and we are done.

REMARK 4.5. Using Theorem 4.4 and the results of $\$ 3$, we can get a *-finite representation of $\hat{\mu}$. However, if the original functional $\Phi$ arises as the integral with 
respect to a measure $\mu$ on $X$, we can do better. The quotient map $\Pi:{ }^{*} X \rightarrow \hat{X}$ will be measure-preserving provided that we restrict $\hat{\mu}$ to the Baire sets.

THEOREM 4.6. Let $(X, \mathscr{B}, \mu)$ be any probability space, $\mathscr{F}$ any algebra of bounded measurable functions containing 1 . If $Q$ is an internal subalgebra of $* \Re$ containing $\left\{{ }^{*} f^{-1}(a, b): f \in \mathscr{F}, a, b \in R\right\}$, and $\mathscr{G}^{\prime}$ is the Baire algebra of $(\hat{X}, \hat{\mathcal{Y}})$, then $\Pi$ : $\left({ }^{*} X, \sigma(\Theta), L\left({ }^{*} \mu\right)\right) \rightarrow\left(\hat{X}, \mathscr{B}^{\prime}, \hat{\mu}\right)$ is measure-preserving.

Proof. Suppose $g \in C(\hat{X})$. Then there is a sequence $f_{1} f_{2}, \ldots, f_{n} \in \mathscr{F}$ such that $\left\|\hat{f}_{n}-g\right\|_{\infty}<1 / n$. If $a, b \in R$,

$$
\begin{aligned}
\Pi^{-1}\left(g^{-1}(a, b)\right) & =\Pi^{-1}\left(\bigcup_{n=1}^{\infty} \hat{f}_{n}^{1}\left(a+\frac{1}{n}, b-\frac{1}{n}\right)\right) \\
& =\bigcup_{n=1}^{\infty} \Pi^{-1}\left(\hat{f}_{n}^{-1}\left(a+\frac{1}{n}, b-\frac{1}{n}\right)\right) \\
& =\bigcup_{n=1}^{\infty} \bigcup_{m=1}^{\infty} * f_{n}^{-1}\left(a+\frac{1}{n}+\frac{1}{m}, b-\frac{1}{n}-\frac{1}{m}\right) \in \sigma(\mathbb{Q}) .
\end{aligned}
$$

Therefore $\left\{B \subset \hat{X}: \Pi^{-1}(B) \in \sigma(\mathscr{Q})\right\}$ is a $\sigma$-algebra containing $g^{-1}(a, b)$ for all $a, b \in R, g \in C(\hat{X})$. Hence it contains $\mathscr{R}^{\prime}$, so $\Pi$ is measurable.

For $B \in \mathscr{G}^{\prime}$, define $\nu(B)=L\left({ }^{*} \mu\right)\left(\Pi^{-1}(B)\right)$. Thus, $\nu$ is a Baire probability measure. Let $g, f_{n}$ be as before.

$$
\begin{aligned}
\int_{\hat{X}} g d \nu & =\lim _{n \rightarrow \infty} \int_{\hat{X}} \hat{f}_{n} d \nu=\lim _{n \rightarrow \infty} \int_{{ }^{*} X} \hat{f}_{n} \circ \Pi d L\left({ }^{*} \mu\right)=\lim _{n \rightarrow \infty} \circ \int_{{ }^{*} X}{ }^{*} f_{n} d^{*} \mu \\
& =\lim _{n \rightarrow \infty} \int_{X} f_{n} d \mu=\lim _{n \rightarrow \infty} \int_{\hat{X}} \hat{f}_{n} d \hat{\mu}=\int_{X} g d \hat{\mu} .
\end{aligned}
$$

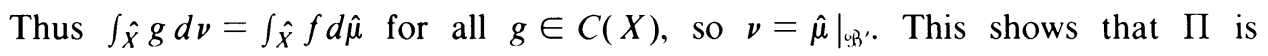
measure-preserving.

REMARK 4.7. As in $\$ 3$, we can establish the existence of a *-finite algebra with the properties required of $\mathcal{Q}$. If, in Theorem 4.6 , we take $\mathscr{F}$ to be the class of all bounded measurable functions on $(X, \mathscr{B}, \mu)$, then $\mathscr{F}$ is closed with respect to the sup norm. Hence $\hat{\mathscr{F}}=C(\hat{X})$. Hence the theory of integration of bounded measurable functions on $X$ is transformed into the theory of integration of continuous functions on $\hat{X}$, and we have $\mathrm{a}^{*}$-finite Loeb representation for this theory. Thus, statements about integration of bounded measurable functions on an arbitrary probability space can be represented as statements about Loeb spaces. What we are doing, of course, is moving from the integration of measurable functions on the original space to integrating continuous functions on the Stone space for $L^{\infty}$. Our construction of $\hat{X}$, the Stone space, is the same as Loeb's [13, p. 77]. We could also take $\mathscr{F}$ to be $C(X)$, provided $X$ is Hausdorff. Then $\hat{\mathscr{F}}=C(\hat{X})$, and again a *-finite Loeb representation results. In a special case, a stronger result holds.

COROllary 4.8. Let $(X, T)$ be compact Hausdorff, $\mu$ a probability measure on the Baire algebra $9 \beta^{\prime}$ on $X$. If $\mathbb{Q}$ is any internal subalgebra of $* \mathrm{G}^{\prime}$ containing $\left\{^{*} f^{-1}(a, b)\right.$ : $f \in C(X), a, b \in R\}$, then st: $\left({ }^{*} X, \sigma(\Theta), L\left({ }^{*} \mu\right)\right) \rightarrow\left(X, G^{\prime}, \mu\right)$ is measure-preserving. 
Proof. $\hat{X}$ is the Stone-Čech compactification of $X$, so we can identify $\hat{X}$ and $X$. Suppose $x \in{ }^{*} X .{ }^{\circ} f(x)=f\left({ }^{\circ} x\right)$ for all $f \in C(X)$. Hence $\Pi(x)={ }^{\circ} x$. Therefore $\Pi=$ st. Using Theorem 4.6, we obtain the desired result.

5. Lifting and pushing down. In this section, we relate measurability properties in internal spaces $(X, \mathcal{Q}, \nu)$, their Loeb spaces $(X, L(\mathbb{Q}), L(\nu))$, and the standard measure spaces which are measure-preserving images of these Loeb spaces. We assume that $(X, Q, \nu)$ satisfies the conditions given in the first paragraph of $\S 2$.

Integration properties in Loeb spaces were studied in $[1, \S 2]$, to which the interested reader is referred. It is shown there that, for $1 \leqslant p<\infty$, there is a nice subspace of ${ }^{*} L^{p}(X, Q, \nu)$ which is isometrically isomorphic via the map $f \rightarrow{ }^{\circ} f$ to $L^{p}(X, L(\mathscr{Q}), L(\nu))$. In this section, we shall concentrate on measurability properties for more general range spaces.

Loeb [15] shows that, if $Y$ is compact metric and $f: X \rightarrow^{*} Y$ is $Q$-measurable, then ${ }^{\circ} f: X \rightarrow Y$ is $L(\mathbb{Q})$-measurable. Conversely, if ${ }^{\circ} \nu(X)<+\infty$ and $g: X \rightarrow R \cup$ $\{-\infty,+\infty\}$ is $L(Q)$-measurable, then there exists $f: X \rightarrow^{*} R, f$ is internal and $Q$-measurable, and ${ }^{\circ} f=g$ almost everywhere. It is these results that we generalize.

Definition 5.1. A topological space $Y$ is regular if:

(i) points are closed;

(ii) for any closed set $A$ and any $x \notin A$, there exist $M$ and $N$ neighborhoods of $A$ and $x$ such that $N \cap M=\varnothing$.

TheOREM 5.2 (Pushing Down). Let ( $Y, \mathcal{T})$ be a regular topological space. Suppose that $(X, \mathcal{Q}, \nu)$ and ${ }^{*} Y$ are taken in a $\kappa$-saturated enlargement, with $\kappa>|\mathcal{T}|$. Suppose ${ }^{\circ} \nu(X)<+\infty$. If $f: X \rightarrow{ }^{*} Y$ is $Q$-measurable and $L(\nu)\left(f^{-1}\left(\mathrm{~ns}\left({ }^{*} Y\right)\right)\right)=L(\nu)(X)$, then ${ }^{\circ} f$ is $L^{\prime}(\mathbb{Q})$-measurable.

Proof. Suppose $A \subset Y$ is closed. Let $\left\{U_{\lambda}\right\}_{\lambda \in \Lambda}$ be the collection of open sets containing $A$. If $y \in Y, y \notin A$, there exist disjoint open $T$ and $M$ with $T \supset A$ and $y \in M$. Therefore $m(y) \cap{ }^{*} T=\varnothing$. Thus $\left(\cap_{\lambda \in \Lambda}{ }^{*} U_{\lambda}\right) \cap \mathrm{ns}\left({ }^{*} Y\right)=\mathrm{st}^{-1}(A)$.

Let $s=\inf \left\{{ }^{\circ} \nu\left(f^{-1}\left({ }^{*} U_{\lambda}\right)\right): \lambda \in \Lambda\right\}$, and suppose $\varepsilon \in R_{+}$. Define $\mathscr{F}_{\lambda}=\{B: B \in$ $\left.* \mathscr{T}, B \subset{ }^{*} U_{\lambda}, \nu\left(f^{-1}(B)\right)>s-\varepsilon\right\}$. Note that ${ }^{*} U_{\lambda} \in \mathscr{F}_{\lambda}$.

If $\lambda_{1}, \ldots, \lambda_{n} \in \Lambda$, then $U_{\lambda_{1}} \cap \cdots \cap U_{\lambda_{n}}$ is an open set containing $A$, so it equals $U_{\lambda}$ for some $\lambda \in \Lambda$. Therefore ${ }^{*} U_{\lambda} \in \mathscr{F}_{\lambda_{1}} \cap \cdots \cap \mathscr{F}_{\lambda_{n}}$, so this last set is not empty. Thus, $\left\{\mathscr{F}_{\lambda}\right\}_{\lambda \in \Lambda}$ has the finite intersection property. By saturation, there exists $B \in \cap_{\lambda} \mathscr{F}_{\lambda}$. That is, $B \in{ }^{*} \mathcal{T}, B \subset \cap_{\lambda}^{*} U_{\lambda}, \nu\left(f^{-1}(B)\right)>s-\varepsilon$. Since $L(\mathbb{Q})$ is complete with respect to $L(\nu), f^{-1}\left(\cap^{*} U_{\lambda}\right) \in L(\mathbb{Q})$. But $f^{-1}\left(\mathrm{~ns}\left({ }^{*} Y\right)\right) \in L(\mathbb{Q})$, so

$$
\begin{aligned}
\left({ }^{\circ} f\right)^{-1}(A) & =f^{-1}\left(\left(\cap{ }^{*} U_{\lambda}\right) \cap \mathrm{ns}\left({ }^{*} Y\right)\right) \\
& =f^{-1}\left(\cap * U_{\lambda}\right) \cap f^{-1}\left(\mathrm{~ns}\left({ }^{*} Y\right)\right) \in L(\mathbb{Q}) .
\end{aligned}
$$

Moreover, ${ }^{\circ} f$ is defined $L(\nu)$-almost everywhere, so ${ }^{\circ} f$ is $L(\mathbb{Q})$-measurable. Since $x \sim y$ implies $f(x)=f(y)$ implies ${ }^{\circ} f(x)={ }^{\circ} f(y),{ }^{\circ} f$ is $L^{\prime}(Q)$-measurable.

TheOrem 5.3 (Lifting). Suppose ${ }^{\circ} \nu(X)<+\infty$ and $Y$ is a Hausdorff space with a countable base of open sets. If $g: X \rightarrow Y$ is $L(\mathbb{Q})$-measurable, there exists an internal $f$ : $X \rightarrow{ }^{*} Y, f$ Q-measurable such that ${ }^{\circ} f(x)=g(x)$ for $L(\nu)$-almost all $x$. In particular, ${ }^{\circ} f$ is $L(\mathbb{Q})$-measurable and has the same distribution as $g$. 
Proof. Let $U_{1}, U_{2}, \ldots$ be a countable base of open sets for $Y$, with $U_{1}=Y$. $g^{-1}\left(U_{n}\right) \in L(Q)$. Therefore we may select $A_{n}^{\prime} \in Q$ such that $L(\nu)\left(A_{n}^{\prime} \triangle g^{-1}\left(U_{n}\right)\right)=0$. We can also take $A_{1}^{\prime}=X$. We want to show there is a function $f_{n}: X \rightarrow{ }^{*} Y$ so that $f_{n}\left(A_{k}^{\prime}\right) \subset{ }^{*} U_{k}$ for $k \leqslant n$. However, we could run into trouble if $\bigcap_{i \in S} U_{i}=\varnothing$ for some $S \subset\{1, \ldots, n\}$, while $\bigcap_{i \in S} A_{i}^{\prime} \neq \varnothing$. We rectify this situation by defining

$$
A_{n}=A_{n}^{\prime}-\bigcup_{\substack{S \subset\{1, \ldots, n\} \\ \cap \in S}} \bigcap_{i \in S} A_{i}^{\prime} .
$$

Note that, if $\cap_{i \in S} U_{i}=\varnothing, \cap_{i \in S} g^{-1}\left(U_{i}\right)=g^{-1}\left(\cap_{i \in S} U_{i}\right)=\varnothing$, so $\nu\left(\cap_{i \in S} A_{i}^{\prime}\right) \simeq 0$. Hence, $\nu\left(A_{n} \triangle A_{n}^{\prime}\right) \simeq 0$, so $L(\nu)\left(A_{n} \triangle g^{-1}\left(U_{n}\right)\right)=0$. For any $S \subset\{1, \ldots, n\}$ for which $\bigcap_{i \in S} U_{i} \neq \varnothing$, select $y_{S} \in \bigcap_{i \in S} U_{i}$. Define $f_{n}(x)=y_{S_{x}}$, where $S_{x}=\{i \leqslant n$ : $\left.x \in A_{i}\right\}$. Note that this defines $f_{n}$ on all of $X$, since $A_{1}=A_{1}^{\prime}=X$ and $\cap_{i \in S} A_{i}=\varnothing$ if $\cap_{i \in S} U_{i}=\varnothing$. Hence, we have found $f_{n}: X \rightarrow{ }^{*} Y, f_{n}$ internal and $Q$-measurable, and $f_{n}\left(A_{k}\right) \subset{ }^{*} U_{k}$ for $k \leqslant n$.

By denumerable comprehension, we may extend $\left\{f_{n}: n \in N\right\},\left\{A_{n}: n \in N\right\}$, and $\left\{{ }^{*} U_{n}: n \in N\right\}$ to internal sequences $\left\{f_{n}: n \in{ }^{*} N\right\},\left\{A_{n}: n \in{ }^{*} N\right\}$, and $\left\{V_{n}: n \in{ }^{*} N\right\}$, where $V_{n}={ }^{*} U_{n}$ for $n \in N$.

$\left\{n\right.$ : $f_{n} Q$-measurable, $\left.k \leqslant n \Rightarrow f_{n}\left(A_{k}\right) \subset V_{k}\right\}$ is internal and contains all $n \in N$; hence it contains some $\omega \in *^{*} N-N$. Let $f=f_{\omega} . f$ is $Q$-measurable.

Thus, for any $k \in N, x \in A_{k} \Rightarrow f(x) \in{ }^{*} U_{k}$. Let $X^{\prime}=X-\cup_{n=1}^{\infty}\left(A_{n} \triangle g^{-1}\left(U_{n}\right)\right)$. Then $X^{\prime} \in L(Q)$, and $L(\nu)\left(X-X^{\prime}\right)=0$. Suppose $x \in X^{\prime}$. Since $x \in$ $\bigcap_{g(x) \in U_{n}} g^{-1}\left(U_{n}\right), \quad x \in \bigcap_{g(x) \in U_{n}} A_{n}$. Therefore $f(x) \in \bigcap_{g(x) \in U_{n}}{ }^{*} U_{n}=m(g(x))$. Thus $x \in X^{\prime}$ implies ${ }^{\circ} f(x)=g(x)$.

REMARK 5.4. In Theorems 3.7, 3.11, and 5.3, the assumption that $Y$ has a countable base excludes many common spaces. This assumption can be weakened as follows. It clearly suffices to assume that there exists a set $B \in L(\Theta), L(\nu)(X-B)$ $=0$, such that $g(B)$ has a countable base in the subspace topology induced by $Y$. In particular, if $Y$ is metric, it suffices to have such a set $B$ with $g(B)$ separable. This will always occur unless measurable cardinals exist. In any case, $g(B)$ will be separable for any reasonable metric space $Y$. For more details, see Billingsley [6, p. 235].

So far, we have considered lifting and pushing down between the internal space $X$ and its Loeb space. Suppose now that $(Z, \mathscr{T}, \mu)$ is any standard measure space, $(X, L(\Theta), L(\nu))$ any Loeb space, and $\Pi: X \rightarrow Z$ any measure-preserving map. In particular, we might be in the situation of $\S 3$, with $X={ }^{*} Z,(Z, \mathscr{B}, \mu)$ Radon, and $\Pi=$ st. We can lift and push down between $X$ and $Z$ in the following way.

Let $(Y, \mathscr{Q})$ be any measurable space and $f: Z \rightarrow Y$ measurable. Then $f \circ \Pi: X \rightarrow Y$ is $L(\Theta)$-measurable, and has the same distribution as $f$. Thus $f \circ \Pi$ is a lifting of $f$ to $(X, L(Q), L(\nu))$. Moreover, if $Y=R \cup\{+\infty,-\infty\}, f \circ \Pi \in L^{p}\left(X, L\left(\Theta^{\prime}\right), L(\nu)\right)$ if and only if $f \in L^{p}(Z, 9, \mu)$.

Pushing down in this situation is possible, at least in a weak sense. Suppose that $g$ : $X \rightarrow Y$ is $\Pi^{-1}(\mathscr{B})$-measurable. Then $g \circ \Pi^{-1}: Z \rightarrow Y$ is $\mathscr{G}$-measurable and has the same distribution as $g$. Theorem 3.11 is a special case of this situation in which $\Pi^{-1}(G)$ has an especially nice form, and where the result is most satisfying. 
But now suppose that $g$ is only $L(Q)$-measurable. If $Y=R \cup\{+\infty,-\infty\}$ and $g \in L^{1}(X, L(\mathscr{Q}), L(\nu))$, and conditional expectation $f=E\left(g \mid \Pi^{-1}(\Re)\right)$ is defined and is $\Pi^{-1}(\Re)$-measurable. Hence $f \circ \Pi^{-1} \in L^{1}(Z, \Re, \mu)$ and has the same distribution as $f$. This gives us a weak push down of $g$ to $Z$ which may prove useful in some situations. Note that the same procedure works if $Y=R^{n}$ or any space in which conditional expectations are defined.

6. Uniform integrability. Uniform integrability is a standard condition on sets of functions. It is useful because it permits a generalization of Lebesgue's Dominated Convergence Theorem. In this section, we shall see that uniform integrability has a simple nonstandard characterization closely related to the integration theory developed by the author in $[1, \S 2]$. This characterization was discovered independently by Loeb and the author; see Loeb's article [28]. We assume throughout that $(X, \mathbb{Q}, \nu)$ satisfies the conditions in the first paragraph of $\$ 2$.

Definition 6.1. Let $\left\{\left(X_{i}, \mathscr{G}_{i}, \mu_{i}\right): i \in I\right\}$ be a family of measure spaces, and $f_{i}$ : $X_{i} \rightarrow R \cup\{+\infty,-\infty\} \Re_{i}$-measurable. We say that $\left\{f_{i}\right\}_{i \in I}$ is uniformly integrable if

(i) $\lim _{\gamma \rightarrow \infty} \sup _{i} \int_{\left|f_{i}\right|>\gamma}\left|f_{i}\right| d \mu_{i}=0$,

(ii) $\sup _{i} \int\left|f_{i}\right| d \mu_{i}<+\infty$,

(iii) $\lim _{\gamma \rightarrow 0} \sup _{i} \int_{\left|f_{i}\right|<\gamma}\left|f_{i}\right| d \mu_{i}=0$.

$\left\{f_{i}\right\}_{i \in I}$ is said to be uniformly $L^{p}$ if $\left\{\left|f_{i}\right|^{p}\right\}$ is uniformly integrable.

REMARK 6.2. If $\sup \mu_{i}\left(X_{i}\right)<+\infty$ and, in particular, if each $X_{i}$ is a probability space, (iii) is automatically satisfied and (i) implies (ii).

Definition 6.3 [1, Definitions 3 and 10]. Suppose $f: X \rightarrow^{*} R$ is Q-measurable. We say that $f$ is $S$-integrable if

(i) $\circ|f| d \nu<+\infty$,

(ii) $A \in \mathbb{Q}, \nu(A) \simeq 0 \Rightarrow \int_{A}|f| d \nu \simeq 0$,

(iii) $A \in \mathbb{Q}, f(A) \subset m(0) \Rightarrow \int_{A}|f| d \nu \simeq 0$.

$f$ is said to be $S L^{p}(1 \leqslant p<\infty)$ if $|f|^{p}$ is $S$-integrable.

REMARK 6.4. If ${ }^{\circ} \nu(X)<+\infty$, (iii) is automatically satisfied; if, in addition, $X$ has no atoms of noninfinitesimal measure, (ii) implies (i). It is shown in [1, Theorem 11] that the map $f \rightarrow{ }^{\circ} f$ determines an isometric isomorphism of $S L^{p}(X, \mathbb{Q}, \nu)$ and $L^{p}(X, L(Q), L(\nu))$.

Theorem 6.5. Suppose $f_{i}:\left(X_{i}, \Re_{i}, \mu_{i}\right) \rightarrow R$. Then $\left\{f_{i}\right\}$ is uniformly $L^{p}(1 \leqslant p<\infty)$ if and only if ${ }^{*} f_{i} \in S L^{p}\left({ }^{*} X_{i},{ }^{*} \Re_{i},{ }^{*} \mu_{i}\right)$ for all $i \in{ }^{*} I$.

Proof. Since $f_{i}$ is $\Re_{i}$-measurable for all $i \in I,{ }^{*} f_{i}$ is $* \mathscr{B}_{i}$-measurable for all $i \in{ }^{*} I$.

Suppose $\left\{f_{i}\right\}$ is uniformly $L^{p}$. Then $\left\{\left|f_{i}\right|^{p}\right\}$ is uniformly integrable. Then there exists $\alpha<\infty$ such that $\sup _{i \in I} \int\left|f_{i}\right|^{p} d \mu_{i}<\alpha$. Hence, for $i \in{ }^{*} I,\left.\left.{ }^{\circ} \int\right|^{*} f_{i}\right|^{p} d^{*} \mu_{i}<\alpha$. Suppose $A \in * \Re_{i},{ }^{*} \mu_{i}(A) \simeq 0$. For each $\gamma \in R_{+}$,

$$
\int_{A}\left|{ }^{*} f_{i}\right|^{p} d^{*} \mu_{i} \leqslant \gamma^{*} \mu_{i}(A)+\int_{\left|f_{i}\right|>\gamma}\left|{ }^{*} f_{i}\right|^{p} d^{*} \mu_{i}
$$

The first term is infinitesimal and the second term tends to zero as $\gamma \rightarrow \infty$; hence 
$\int_{A}\left|{ }^{*} f_{i}\right|^{p} d^{*} \mu_{i} \simeq 0$. Suppose $A \in *^{*} \mathscr{B}_{i},{ }^{*} f_{i}(A) \subset m(0)$. For each $\gamma \in R_{+}$,

$$
\int_{A}\left|f_{i}\right|^{p} d^{*} \mu_{i} \rightarrow \int_{\left|f_{i}\right|^{p}<\gamma}\left|{ }^{*} f_{i}\right|^{p} d^{*} \mu_{i} \rightarrow 0 \quad \text { as } \gamma \rightarrow 0
$$

Hence $\int_{A}\left|{ }^{*} f_{i}\right|^{p} d^{*} \mu_{i} \simeq 0$. Thus, $\left\{f_{i}\right\}$ uniformly $L^{p}$ implies ${ }^{*} f_{i} \in S L^{p}$ for all $i \in{ }^{*} I$.

Conversely, suppose ${ }^{*} f_{i} \in S L^{p}$ for each $i \in{ }^{*} I$. Then $\sup _{i \in *_{I}} \int\left|{ }^{*} f_{i}\right|^{p} d^{*} \mu_{i}$ is less than any infinite $\gamma$, so it must be finite. Hence $\left.\left.\sup _{i \in I} \int\right|^{*} f_{i}\right|^{p} d^{*} \mu_{i}<+\infty$. Suppose $\gamma \in{ }^{*} R_{+}$. Let $A_{i}=\left\{x \in{ }^{*} X_{i}:\left|f_{i}(x)\right|^{p}>\gamma\right\}$. If $\gamma$ is infinite, ${ }^{*} \mu_{i}\left(A_{i}\right) \simeq 0$, so $\left.\left.\int_{A_{i}}\right|^{*} f_{i}\right|^{p} d^{*} \mu_{i} \simeq 0$. Therefore $\left.\left.\sup _{i \in *_{I}} \int_{A_{i}}\right|^{*} f_{i}\right|^{p} d^{*} \mu_{i} \simeq 0$. Thus

$$
\lim _{\gamma \rightarrow \infty} \sup _{i \in I} \int_{A_{i}}\left|{ }^{*} f_{i}\right|^{p} d^{*} \mu_{i}=0
$$

Essentially the same argument shows that

$$
\lim _{\substack{\gamma \rightarrow 0 \\ \gamma \in R_{+}}} \sup _{i \in I} \int_{\left|f_{i}\right|^{p}<\gamma}\left|f_{i}\right|^{p} d^{*} \mu_{i}=0 .
$$

Hence ${ }^{*} f_{i} \in S L^{p}$ for all $i \in{ }^{*} I$ implies $\left\{f_{i}\right\}_{i \in I}$ is uniformly $L^{p}$.

Corollary 6.6. Suppose $f \in L^{p}(X, \Re, \mu)$. Then ${ }^{*} f \in S L^{p}\left({ }^{*} X,{ }^{*} \Re,{ }^{*} \mu\right)(1 \leqslant p<$ $\infty)$.

Remark 6.7. The definition of $S L^{p}$ does not generalize readily to $p=\infty$; it seems that each possible definition has some undesirable properties. Some thoughts on this question are given in \$III.8 of Anderson [2].

7. Conditional expectations and martingales. Conditional expectations and martingales are important tools in probability theory. Brownian motion and Itô integrals, particular examples of martingales, were treated in [1]. We shall see that general martingales can be represented naturally in *-finite spaces. Lindstrom [26] and Hoover and Perkins [25] have recently developed the nonstandard theory of stochastic integration with respect to local martingales. The appropriate notion of representation of martingales in the context of stochastic integration involves path properties which are not considered here. Thus, readers interested in stochastic integration should consult Lindstrom [26] and Hoover and Perkins [25] rather than this section. The theorems we give are considerably simpler, and may still be of interest for purposes other than stochastic integration.

Suppose $Q^{\prime}$ is a ${ }^{*}$-finite subalgebra of $Q$. Then there exists a ${ }^{*}$-finite partition $\mathscr{P} \subset \mathbb{Q}^{\prime}$ which generates $\mathbb{Q}^{\prime}$. In other words, $E \in \mathbb{Q}^{\prime} \Leftrightarrow E=\cup_{x \in E} P_{x}$ where $P_{x}$ is the element of 9 containing $x$.

Suppose more generally that $\mathbb{Q}^{\prime}$ is the ${ }^{*}$ - $\sigma$-algebra generated by a *-countable partition of $X$. If $f \in{ }^{*} L^{1}(X, \mathbb{Q}, \nu)$ the conditional expectation is given by

$$
E\left(f \mid Q^{\prime}\right)(x)=\frac{1}{\nu\left(P_{x}\right)} \int_{P_{x}} f d \nu .
$$

Note that $\nu\left(\cup_{\nu\left(P_{1}\right)=0} P_{x}\right)=0$. 


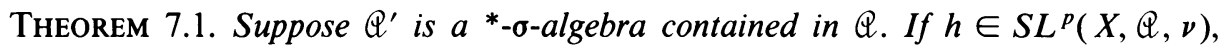
then $E\left(h \mid \mathbb{Q}^{\prime}\right) \in S L^{p}\left(X, \mathbb{Q}^{\prime}, \nu\right)$ and ${ }^{\circ} E\left(h \mid \mathbb{Q}^{\prime}\right)=E\left({ }^{\circ} h \mid L\left(\mathbb{Q}^{\prime}\right)\right)=E\left({ }^{\circ} h \mid L^{\prime}\left(\mathbb{Q}^{\prime}\right)\right)$ $L\left(\left.\nu\right|_{\mathbb{Q}^{\prime}}\right)$-almost everywhere.

Proof. This is proved in [1, Theorem 12(ii)] in the special case that $\mathbb{Q}^{\prime}$ is *-finite. The same proof works if $Q^{\prime}$ is the ${ }^{*}$ - $\sigma$-algebra generated by a ${ }^{*}$-countable partition of $X$.

Let $g=E\left(h \mid Q^{\prime}\right)$, and $\omega \in{ }^{*} N-N$. Let $Q^{\prime \prime}$ be the ${ }^{*}$ - $\sigma$-algebra generated by the *-countable partition $\left\{g^{-1}([i / \omega,(i+1) / \omega)): i \in{ }^{*} Z\right\}$. Thus $E\left(h \mid \mathbb{Q}^{\prime \prime}\right) \in$ $S L^{p}\left(X, \mathbb{Q}^{\prime \prime}, \nu\right)$. But $\left|g(x)-E\left(h \mid \mathbb{Q}^{\prime \prime}\right)(x)\right|<1 / \omega \simeq 0$ for all $x \in X$, so $g \in$ $S L^{p}\left(X, Q^{\prime}, \nu\right)$. The same argument used in [1, Theorem 12(ii)] shows that ${ }^{\circ} g=$ $E\left({ }^{\circ} h \mid L\left(\mathbb{Q}^{\prime}\right)\right)$. But ${ }^{\circ} g$ is $L^{\prime}\left(\mathbb{Q}^{\prime}\right)$-measurable and $L^{\prime}\left(\mathbb{Q}^{\prime}\right) \subset L\left(\mathbb{Q}^{\prime}\right)$, so

$$
{ }^{\circ} g=E\left({ }^{\circ} h \mid L^{\prime}\left(Q^{\prime}\right)\right) \text {. }
$$

Definition 7.2. Let $(Z, \mathcal{C}, \mu)$ be a probability space. Let $I$ be a directed set, called the set of time parameters. Let $\left\{\widetilde{F}_{t}\right\}_{t \in I}$ be an increasing family of $\sigma$-algebras contained in $\mathcal{C}$ (i.e., $s<t \Rightarrow \mathscr{F}_{s} \subset \mathscr{F}_{t}$ ). We say that $M$ is an $\left\{\mathscr{F}_{t}\right\}$-martingale if $M$ is a collection $\left\{M_{t}\right\}_{t \in I} \subset L^{1}(Z, \bigodot, \mu)$ satisfying $E\left(M_{t} \mid \mathscr{F}_{s}\right)=M_{s}$ whenever $s<t$. $M$ is said to be uniformly $L^{p}$ if $\left\{M_{t}\right\}_{t \in I}$ is uniformly $L^{p}$. Two martingales $M$ and $M^{\prime}$ are said to have the same finite-dimensional distributions if, for any $t_{1}, \ldots, t_{n} \in I$ and $a_{1}, \ldots, a_{n} \in R(n \in N)$,

$$
\mu\left(\left\{z: M_{t_{i}}(z)<a_{i}, 1 \leqslant i \leqslant n\right\}\right)=\mu\left(\left\{z: M_{t_{i}}^{\prime}(z)<a_{i}, 1 \leqslant i \leqslant n\right\}\right) .
$$

Any element of the nonstandard extension of the class of martingales is called a ${ }^{*}$-martingale. A *-martingale $M$ is called *-finite if there is a ${ }^{*}$-finite algebra $Q$ such that every $M_{t}$ is $Q$-measurable and such that, for any internal totally ordered set $J$ of time parameters, there is a ${ }^{*}$-finite collection $-\infty=t_{1}<t_{2}<\cdots<t_{\omega}=+\infty \subset J$ $\cup\{-\infty,+\infty\}$ such that $M_{t}=M_{s}$ whenever $s, t \in\left(t_{i}, t_{i+1}\right)$. If $I$ is any partially ordered set, $t \in{ }^{*} I$ is said to be finite if there exists $s \in I, s \geqslant t$.

We shall first study the relationship between the *-martingales on $(X, \mathcal{Q}, \nu)$ and the martingales on $\left(X, L^{\prime}(Q), L(\nu)\right)$. The uniform integrability condition in the following theorem was pointed out by K. D. Stroyan.

Theorem 7.3 (Pushing Down for MARTINgales). Suppose $I$ is an internal directed set, $\left\{Q_{t}\right\}_{t \in I}$ an internal increasing family of ${ }^{*}-\sigma$-algebras with $Q_{t} \subset \mathbb{Q}$ for all $t$. If $Y$ is an $\left\{Q_{t}\right\}^{-*}$-martingale and $Y_{t} \in S L^{1}(X, \mathbb{Q}, \nu)$ for all $t \in I$, then ${ }^{\circ} Y$ is an $\left\{L^{\prime}\left(\mathbb{Q}_{t}\right)\right\}$ - and an $\left\{L\left(Q_{t}\right)\right\}$-uniformly integrable martingale.

Proof. First note that $\left\{L^{\prime}\left(Q_{t}\right)\right\}$ is an increasing family of $\sigma$-algebras contained in $L^{\prime}(\mathbb{Q})$. Since $Y_{t} \in S L^{1}(X, \mathbb{Q}, \nu),{ }^{\circ} Y_{t} \in L^{1}\left(X, L^{\prime}(\mathbb{Q}), L(\nu)\right)$ by [1, Theorem 9]. If $s<t, E\left({ }^{\circ} Y_{t} \mid L^{\prime}\left(\mathbb{Q}_{s}\right)\right)={ }^{\circ} E\left(Y_{t} \mid Q_{s}\right)={ }^{\circ} Y_{s}$ by Theorem 7.1. Hence ${ }^{\circ} Y$ is an $\left\{L^{\prime}\left(Q_{t}\right)\right\}-$ martingale. The argument for $\left\{L\left(Q_{t}\right)\right\}$ is the same. If $\gamma$ is infinite,

$$
\sup _{t \in I} \int_{\left|Y_{t}\right|>\gamma}\left|Y_{t}\right| d \nu \simeq 0
$$

since $I$ is internal. Hence $\lim _{\gamma \rightarrow \infty} \sup _{t \in I} \int_{\left|Y_{t}\right|>\gamma}\left|Y_{t}\right| d \nu=0$. Since $\nu(X)=1,\left\{{ }^{\circ} Y_{t}\right\}$ is uniformly integrable. 
TheOREM 7.4 (LifTING FOR MARTINGALES). Suppose that $I$ is a directed set and $\left\{\Theta_{t}\right\}_{t \in I}$ is an increasing family of ${ }^{*}$ - $\sigma$-algebras contained in $Q$. Let $M$ be a uniformly integrable $\left\{L^{\prime}\left(\mathbb{Q}_{t}\right)\right\}$ - or $\left\{L\left(\mathbb{Q}_{t}\right)\right\}$-martingale.

(i) If $I$ and the map $t \rightarrow Q_{t}$ are internal, there is an $\left\{\mathbb{Q}_{t}\right\}-^{*}-$ martingale $Y$ such that ${ }^{\circ} Y_{t}=M_{t} L\left(\left.\nu\right|_{\mathbb{Q}_{t}}\right)$-almost everywhere.

(ii) If $X$ and the elements of I are taken in a $\kappa$-saturated model and $|I|<\kappa$, there is a ${ }^{*}$-finite internal directed set $J \supset I$, an increasing family of ${ }^{*}-\sigma$-algebras $\left\{\Theta_{t}\right\}_{t \in J} \subset \mathbb{A}$, and an $\left\{\mathbb{Q}_{t}\right\}-{ }^{*}$-martingale $Y$ such that, for $t \in I,{ }^{\circ} Y_{t}=M_{t} L\left(\left.\nu\right|_{\mathbb{Q}_{t}}\right)$-almost everywhere.

Proof. We shall give the proof for $M$ an $\left\{L\left(Q_{t}\right)\right\}$-martingale. The proof for an $\left\{L^{\prime}\left(Q_{t}\right)\right\}$-martingale is the same.

(i) Note that $I$ is a set in standard set theory. Hence $I$ is a standard directed set. Since $M$ is uniformly integrable, there exists $M_{\infty} \in L^{1}(X, L(\Theta), L(\nu))$ such that $M_{t}=E\left(M_{\infty} \mid L\left(Q_{t}\right)\right)$ by the Mean Martingale Convergence Theorem (Helms [8]).

By [1, Theorem 11(ii)], there exists $Y_{\infty} \in S L^{1}(X, Q, \nu)$ such that ${ }^{\circ} Y_{\infty}=M L(\nu)$ almost everywhere. Define $Y_{t}=E\left(Y_{\infty} \mid Q_{t}\right)$. Then $Y$ is an $\left\{Q_{t}\right\}-{ }^{*}$-martingale. By Theorem 7.1,

$$
{ }^{\circ} Y_{t}={ }^{\circ} E\left(Y_{\infty} \mid Q_{t}\right)=E\left({ }^{\circ} Y_{\infty} \mid L\left(Q_{t}\right)\right)=E\left(M_{\infty} \mid L\left(Q_{t}\right)\right)=M_{t}
$$

$L\left(\left.\nu\right|_{\mathbb{Q}_{t}}\right)$-almost everywhere.

(ii) Let $\mathcal{G}=\left\{(J, \xi): J\right.$ is a ${ }^{*}$-finite internal directed set, $\left\{\xi_{j}\right\}_{j \in J}$ is an internal increasing family of ${ }^{*}-\sigma$-algebras contained in $\left.\mathcal{Q}\right\}$. For $t \in I$, let $\mathcal{G}_{t}=\{(J, \xi) \in \mathcal{G}$ : $\left.t \in J, \xi_{t}=\mathbb{Q}_{t}\right\}$. If $n \in N$ and $t_{1}, \ldots, t_{n} \in I$,

$$
\left(\left\{t_{1}, \ldots, t_{n}\right\}, \xi_{t_{i}}=\mathcal{Q}_{t_{i}}\right) \in \mathcal{S}_{t_{i}} \cap \cdots \cap \mathcal{S}_{t_{n}},
$$

so this intersection is not empty. By saturation, there exists some $(J, \xi) \in \cap_{t \in I} \mathcal{G}_{t}$. Define $Q_{t}=\xi_{t}$ for $t \in J$. Thus, $J$ is *-finite and $\left\{Q_{t}\right\}$ is an internal increasing family of ${ }^{*}$ - $\sigma$-algebras contained in $Q$.

As in (i), there exists $M_{\infty} \in L^{1}(X, L(Q), L(\nu))$ such that $E\left(M_{\infty} \mid L\left(Q_{t}\right)\right)=M_{t}$ for each $t \in I$. For any $t \in J$, define $N_{t}=E\left(M_{\infty} \mid L\left(Q_{t}\right)\right)$. With this extension, $J$, $\left\{Q_{t}\right\}_{t \in J}$, and $\left\{N_{t}\right\}_{t \in J}$ satisfy the hypotheses of (i). Hence there is an internal $\mathbb{Q}_{t^{-}}{ }^{*}$-martingale $Y$ such that, for all $t \in J,{ }^{\circ} Y_{t}=N_{t} L\left(\left.\nu\right|_{Q_{t}}\right)$-almost everywhere. Thus, for $t \in I,{ }^{\circ} Y_{t}=M_{t} L\left(\left.\nu\right|_{Q_{t}}\right)$-almost everywhere.

Now we turn to the problem of representing arbitrary standard martingales. The representation will take a strong form if the underlying probability space is Radon. The neat construction for obtaining the algebras $Q_{t}$ in the proof is due to Loeb.

THEOREM 7.5. Let $(Z, e, \mu)$ be any probability space. If $M$ is any $\left\{\widetilde{\mathscr{F}}_{t}\right\}_{t \in I}$-martingale, there is $a{ }^{*}$-finite martingale $Y$ such that, for each $t \in I, \int\left|Y_{t}-{ }^{*} M_{t}\right| d^{*} \mu \simeq 0$. In particular, $\left.{ }^{\circ} Y\right|_{I}$ and $M$ have the same finite-dimensional distributions.

Proof. Find a ${ }^{*}$-finite algebra $\mathscr{Q}$ such that $\left\{{ }^{*} C: C \in \mathcal{C}\right\} \subset \mathbb{Q} \subset{ }^{*} \mathcal{C}$. For $t \in{ }^{*} I$, let

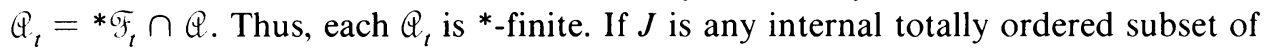
$I,\left\{Q_{t}: t \in J\right\}$ is a totally ordered class of ${ }^{*}$-subalgebras of $Q$. Since $\Theta^{*}$ is ${ }^{*}$-finite, there are only ${ }^{*}$-finitely many such ${ }^{*}$-subalgebras. Hence there exist $-\infty=t_{1}<$ $\cdots<t_{\omega}=+\infty \in J \cup\{-\infty,+\infty\}\left(\omega \in{ }^{*} N\right)$ such that $\mathbb{Q}_{s}=\mathbb{Q}_{t}$ whenever $s, t \in$ $\left(t_{i}, t_{i+1}\right)$. 
Let $Y_{t}=E\left({ }^{*} M_{t} \mid Q_{t}\right)$. If $s<t$,

$$
\begin{aligned}
Y_{s} & =E\left({ }^{*} M_{s} \mid Q_{s}\right)=E\left(E\left({ }^{*} M_{t} \mid * \mathscr{F}_{s}\right) \mid Q_{s}\right)=E\left({ }^{*} M_{t} \mid Q_{s}\right) \\
& =E\left(E\left(* M_{t} \mid Q_{t}\right) \mid Q_{s}\right)=E\left(Y_{t} \mid Q_{s}\right) .
\end{aligned}
$$

Hence $\left\{Y_{t}\right\}_{t \in *_{I}}$ is an $\left\{\mathbb{Q}_{t}\right\}^{-*}$-martingale.

If $t_{1}, \ldots, t_{\omega}$ are as above, and $s, t \in\left(t_{i}, t_{i+1}\right), s<t$,

$$
Y_{s}=E\left(Y_{t} \mid Q_{s}\right)=E\left(Y_{t} \mid Q_{t}\right)=Y_{t}
$$

Since $\mathscr{Q}$ is also *-finite, $Y$ is a ${ }^{*}$-finite martingale.

Now suppose $t \in I . M_{t} \in L^{1}(Z, \mathcal{C}, \mu)$, so ${ }^{*} M_{t} \in S L^{1}\left({ }^{*} Z,{ }^{*} \complement,{ }^{*} \mu\right)$ by Corollary 6.6. Hence $Y_{t} \in S L^{1}$ by Theorem 7.1. Therefore $\int\left|Y_{t}-{ }^{*} M_{t}\right| d^{*} \mu \simeq \int^{\circ}\left|Y_{t}-{ }^{*} M_{t}\right| d L\left({ }^{*} \mu\right)$ by [2, Theorem 9]. But $Y_{t}=E\left({ }^{*} M_{t} \mid Q_{t}\right)$ and ${ }^{*} M_{t}^{-1}(a, b) \in{ }^{*} F_{t} \cap \mathbb{Q}=Q_{t}$ for all $a, b \in R$. Thus $Y_{t}(z) \simeq{ }^{*} M_{t}(z)$ for $L\left({ }^{*} \mu\right)$-almost all $z$ (namely all $z$ for which ${ }^{*} M_{t}(z)$ is finite). Thus ${ }^{\circ}\left|Y_{t}-{ }^{*} M_{t}\right|$ is zero almost everywhere, so $\int\left|Y_{t}-{ }^{*} M_{t}\right| d^{*} \mu$ $\simeq 0$.

Thus $\left.{ }^{\circ} Y\right|_{I}$ and $\left.{ }^{\circ *} M\right|_{I}$ have the same finite-dimensional distributions. Using the Transfer Principle, it is easy to see that $\left.{ }^{\circ *} M\right|_{I}$ and $M$ have the same finite-dimensional distributions. Thus $\left.{ }^{\circ} Y\right|_{I}$ and $M$ have the same finite-dimensional distributions.

THEOREM 7.6. Let $(Z, \Re, \mu)$ be Radon with completion $(Z, \mathcal{C}, \mu)$. Suppose $\left\{\mathscr{F}_{t}\right\}_{t \in I}$ is an increasing family of $\sigma$-algebras contained in $\mathcal{C}$. If $M$ is an $\left\{\mathscr{F}_{t}\right\}$-martingale, there is a ${ }^{*}$-finite martingale $\left\{Y_{t}\right\}_{t \in *_{I}}$ on ${ }^{*} Z$ such that, for all $t \in I, Y_{t}=M_{t} \circ$ st $L\left({ }^{*} \mu\right)$-almost everywhere, and st is measure-preserving.

Proof. Form $Q_{t}$ and $Y$ as in the proof of Theorem 7.5. Since $Q \supset\left\{{ }^{*} C: C \in \mathcal{C}\right\}$, it is $S$-separating. Hence st: $\left({ }^{*} Z, L^{\prime}(\mathbb{Q}), L\left({ }^{*} \mu\right)\right) \rightarrow(Z, \mathcal{C}, \mu)$ is measure-preserving by Theorem 3.3. For any $t \in I, M_{t}$ is $\mathscr{F}_{t}$-measurable, so ${ }^{\circ *} M_{t}=M_{t} \circ$ st $L\left({ }^{*} \mu\right)$-almost everywhere by Theorem 3.7. But ${ }^{\circ} Y_{t}={ }^{\circ}{ }^{*} M_{t}$ almost everywhere by Theorem 7.5, so ${ }^{\circ} Y_{t}=M_{t} \circ$ st almost everywhere.

REMARK 7.7. We could replace ${ }^{*} Z$ by the ${ }^{*}$-finite space ${ }^{*} Z / \sim$ as in Corollary 3.4.

8. Standardly distributed measures. Let $X$ be a Hausdorff space, and $\nu$ an internal nonnegative Borel measure on ${ }^{*} X,{ }^{\circ} \nu\left({ }^{*} X\right)<\infty . \nu$ is said to be near-standardly concentrated if $L(\nu)\left(\mathrm{st}^{-1}(X)\right)={ }^{\circ} \nu\left({ }^{*} X\right)$. This property is intimately related to weak convergence of measures, as discussed in Anderson and Rashid [4].

In this section, we discuss a stronger property of measures. The study is motivated by an application to core theory in mathematical economics (Anderson [3]), but it may well have application to other problems. In Theorem 8.7, we shall see that measures obtained by sampling with replacement have this property.

DeFinition 8.1. We say $\nu$ is standardly distributed if, for all standard Borel sets $B \subset X, \nu\left({ }^{*} B\right) \simeq L(\nu)\left(\mathrm{st}^{-1}(B)\right)$.

There are a number of natural examples of standardly distributed measures. The first two-those with finite standard support and the stars of standard measuresclearly have many special properties. However, most of these special properties carry over to all standardly distributed measures, so these examples may be regarded as prototypical. 
EXAmple 8.2. Suppose $B$ is a finite or countable set of standard points, and $\nu$ is concentrated on $B$ (i.e., $\nu\left({ }^{*} X-B\right) \simeq 0$ ). For any standard Borel set $A,{ }^{*} A \cap B=$ $\mathrm{st}^{-1}(A) \cap B$, so

$$
\nu\left(*^{*} A\right) \simeq L(\nu)\left({ }^{*} A\right)=L(\nu)\left(*^{*} A \cap B\right)=L(\nu)\left(\mathrm{st}^{-1}(A) \cap B\right)=L(\nu)\left(\mathrm{st}^{-1}(A)\right),
$$

so $\nu$ is standardly distributed.

EXAmple 8.3. Let $\mu$ be a Radon measure on $X$. For any Borel $B,{ }^{*} \mu\left({ }^{*} B\right)=\mu(B)$ $=L\left({ }^{*} \mu\right)\left(\mathrm{st}^{-1}(B)\right)$ by Theorem 3.3 , so ${ }^{*} \mu$ is standardly distributed.

I am grateful to the referee for pointing out an error in part (ii) of the following proposition, and for pointing out that part (i) follows (at least in the compact Hausdorff case) from Corollary 3 of Loeb [27].

Proposition 8.4. (i) Suppose $\nu\left({ }^{*} B\right) \simeq \mu(B)$ for all Borel $B$, where $\mu$ is a Radon measure. Then $\nu$ is standardly distributed, and $\mu(B)=L(\nu)\left(\mathrm{st}^{-1}(B)\right)$ for all Borel $B$.

(ii) Suppose $\nu$ is standardly distributed. If we define $\mu(B)=L(\nu)\left(\mathrm{st}^{-1}(B)\right)$ for Baire $B$, then $\nu$ is in the weak-star monad of $\mu$, i.e. $\int_{*}{ }^{*} f d \nu \simeq \int_{X} f d \mu$ for all bounded continuous $f: X \rightarrow R$. If $X$ is completely regular, $\mu(B)=L(\nu)\left(\mathrm{st}^{-1}(B)\right)$ is defined for all Borel $B$, and $\mu$ is a Radon measure.

Proof. If $K \subset X$ is compact,

$$
\mu(K) \simeq \nu\left({ }^{*} K\right) \simeq L(\nu)\left({ }^{*} K\right) \leqslant L(\nu)\left(\mathrm{st}^{-1}(K)\right),
$$

so $\mu(K) \leqslant L(\nu)\left(\right.$ st $\left.^{-1}(K)\right)$. If $O \subset X$ is open,

$$
\mu(O) \simeq \nu(* O) \simeq L(\nu)(* O) \geqslant L(\nu)\left(\mathrm{st}^{-1}(O)\right)
$$

so $\mu(O) \geqslant L(\nu)\left(\right.$ st $\left.^{-1}(O)\right)$.

For any Borel set $B$ and $\varepsilon>0$, we can find $K$ compact and open so that $K \subset B \subset O, \mu(O \backslash K)<\varepsilon$, since $\mu$ is Radon. Hence

$$
\mu(K) \leqslant L(\nu)\left(\mathrm{st}^{-1}(K)\right) \leqslant L(\nu)\left(\mathrm{st}^{-1}(B)\right) \leqslant L(\nu)\left(\mathrm{st}^{-1}(O)\right) \leqslant \mu(O),
$$

so $L(\nu)\left(\mathrm{st}^{-1}(B)\right)=\mu(B) \simeq \nu\left({ }^{*} B\right)$, so $\nu$ is standardly distributed.

To prove (ii), note $\nu\left({ }^{*} X\right) \simeq L(\nu)\left(\operatorname{st}^{-1}(X)\right)$, so $L(\nu)\left({ }^{*} X-\mathrm{st}^{-1}(X)\right)=0$. By Anderson and Rashid [4, Lemma 2], $\mu$ is defined for Baire $B$ and $\nu$ is in its weak-star monad. If $X$ is completely regular, then by Loeb [27, Theorem 3], $\mu$ is defined for Borel $B$ and is the unique regular extension of its values on Baire sets; since it is also tight, it is Radon.

EXAMPLE 8.5. Bernstein and Wattenberg [5] showed there is a *finite set $F$ so that, defining $\nu(A)=|F \cap A| /|F|$ for internal $A, \nu\left({ }^{*} B\right)$ is infinitely close to the Lebesgue measure of $B$ for all Lebesgue subsets of $[0,1]$, and they used this as a basis for a nonstandard integration theory. The preceding proposition shows $\nu$ is standardly distributed. It also shows that the internal standardly distibuted $\nu$ are exactly the internal measures which represent Radon measures in the Bernstein-Wattenberg style $\left(\nu\left({ }^{*} B\right) \simeq \mu(B)\right)$. This also shows there are standardly distributed measures which are not infinitely close (in the total variation norm) to standard measures.

EXAMPLE 8.6. This example shows the converse of part (ii) of Proposition 8.4 is false. Let $\mu$ be point mass at $0, \nu$ point mass at $\varepsilon$ for some $0 \neq \varepsilon \simeq 0$. $L(\nu) \mathrm{st}^{-1}=\mu$, but $\nu(*\{0\})=0 \neq \mu(\{0\})$. 
The following theorem shows that measures obtained by sampling with replacement are almost surely standardly distributed. It is related in spirit to Keisler's Elementary Chain Theorem [11, Theorem 4.11] and is the main reason the concept is interesting. Part (iii) is an analogue of the Glivenko-Cantelli theorem (Billingsley [6, p. 103]). Part (i) strengthens a result of Wattenberg [29, Theorem II.4].

THEOREM 8.7. Let $\mu$ be a Radon probability measure on $X$ and assume our nonstandard model is $\kappa^{+}$-saturated where $\kappa$ is the cardinality of the Borel algebra. Let $\Omega={ }^{*}\left(\Pi_{n \in N} X\right)$, endowed with the internal probability measure $P$ of sampling with replacement (i.e., $P$ is the ${ }^{*}$-countable product of copies of ${ }^{*} \mu$ ). For $\omega \in \Omega$ and $n \in{ }^{*} N$, let $\nu_{\omega}^{n}(A)=\left|A \cap\left\{\omega_{1}, \ldots, \omega_{n}\right\}\right| / n$, for all internal Borel $A \subset{ }^{*} X$ (i.e., $\nu_{\omega}^{n}$ is the measure induced by the first $n$ samples).

(i) Given $n$ infinite $\left(\nu_{\omega}^{n}\right.$ is standardly distributed and $L\left(\nu_{\omega}^{n}\right) \mathrm{st}^{-1}(B)=(B)$ for all Borel $B$ ) for $L(P)$-almost all $\omega$.

(ii) Let $M$ be any internal set of measures containing all the standardly distributed measures $\nu$ satisfying $L(\nu) \mathrm{st}^{-1}=\mu$. For $L(P)$-almost all $\omega, \nu_{\omega}^{n} \in M$ for all infinite $n$.

(iii) If $X$ is separable metric, then for $L(P)$-almost all $\omega, L\left(\nu_{\omega}^{n}\right) \mathrm{st}^{-1}=\mu$ for all infinite $n$.

Proof. Fix an internal Borel set $B . n\left(\nu_{.}^{n}(B)-{ }^{*} \mu(B)\right)$ is the sum of $n$ independent random variables with mean 0 and variance ${ }^{*} \mu(B)(1-* \mu(B))<1$. If $n$ is infinite, Bernstein's Inequality (Keilser [11, Theorem 4.3]) shows

$$
P\left(\left|\nu^{n}(B)-{ }^{*} \mu(B)\right|>n^{-1 / 4}\right) \leqslant 2 e^{-\sqrt{n} / 4} .
$$

Now fix $n$ infinite. By the saturation assumption, we can find a collection of $n$ internal Borel sets containing ${ }^{*} B$ for every standard Borel set $B$. Thus, we can find an internal $\Omega_{n}^{\prime} \subset \Omega, P\left(\Omega_{n}^{\prime}\right) \geqslant 1-2 n e^{-\sqrt{n} / 4}, \nu_{\omega}^{n}\left({ }^{*} B\right) \simeq \mu(B)$ for all standard Borel $B$ and hence (i) follows from Proposition 8.4(i).

$\omega \in \Omega_{n}^{\prime} \Rightarrow \nu_{\omega}^{n} \in M$. For each infinite $n,\left\{\omega: \nu_{\omega}^{m} \in M\right.$ for all $\left.m \geqslant n\right\}$ is internal and has probability at least $1-\sum_{m \geqslant n} 2 m e^{-\sqrt{m} / 4}$. Since $M$ is internal,

$$
P\left(\left\{\omega: \nu_{\omega}^{m} \in M \text { for all } m \geqslant n\right\}\right) \geqslant 1-\sum_{m \geqslant n} 2 m e^{-\sqrt{m} / 4}
$$

holds for some finite $n$ as well. Hence

$$
L(P)\left(\left\{\omega: \nu_{\omega}^{m} \in M \text { for all infinite } m\right\}\right)=1-\lim _{n \rightarrow \infty} \sum_{m \geqslant n} 2 m e^{-\sqrt{m} / 4}=1,
$$

proving (ii).

(iii) Suppose $X$ is separable metric. Then (Billingsley [6, p. 239]) the set of Borel probability measures on $X$ is also metrizable, with metric denoted by $\rho$. Given $\varepsilon>0$, let $M_{\varepsilon}=*\{\nu: \rho(\nu, \mu)<\varepsilon\}$. If $L(\nu) \mathrm{st}^{-1}=\mu,{ }^{*} \rho\left(\nu,{ }^{*} \mu\right) \simeq 0$, so $M_{\varepsilon}$ satisfies the hypotheses of (ii). Hence, for $L(P)$-almost all $\omega, \nu_{\omega}^{n} \in M_{\varepsilon}$ for all infinite $n$. Therefore, for $L(P)$-almost all $\omega, \nu_{\omega}^{n} \in \bigcap_{\varepsilon>0, \varepsilon \in R} M_{\varepsilon}$ for all infinite $n$, i.e., ${ }^{*} \rho\left(\nu_{\omega}^{n},{ }^{*} \mu\right)$ $\simeq 0$, or $L\left(\nu^{n}\right) \mathrm{st}^{-1}=\mu$ (Anderson and Rashid [4, Lemma 2]) as Baire measures; but all Borel sets are Baire sets (Billingsley [6]).

REMARK 8.8. The assumption that $X$ is separable in part (iii) is only needed to avoid pathologies which could arise if measurable cardinals exist. The actual 
condition required is that every separable subset of $X$ have nonmeasurable cardinal (Billingsley [6, p. 239]).

EXAMPLE 8.9. One might suspect that, for $L(P)$-almost all $\omega, \nu_{\omega}^{n}$ is standardly distributed for all infinite $n$. This example shows that the situation is quite different. Let $\mu$ be Lebesgue measure on $[0,1]$. Given $\omega$, let

$$
B_{\omega}=\bigcup_{n \in N}\left({ }^{\circ} \omega_{n}-1 / 2^{n+2},{ }^{\circ} \omega_{n}+1 / 2^{n+2}\right) .
$$

Then $\mu\left(B_{\omega}\right) \leqslant \frac{1}{2}$. Hence, for $L(P)$-almost all $\omega, L\left(\nu_{\omega}^{n}\right)\left(\mathrm{st}^{-1}\left(B_{\omega}\right)\right)=\mu\left(B_{\omega}\right) \leqslant \frac{1}{2}$ for all infinite $n$ by Theorem 8.7(iii). However, if $n$ is finite, $\nu_{\omega}^{n}\left({ }^{*} B_{\omega}\right)=1$. We conclude there exists $n$ infinite (depending on $\omega)$ such that $\nu_{\omega}^{n}\left({ }^{*} B_{\omega}\right)=1 \nsucc L\left(\nu_{\omega}^{n}\right)\left(\operatorname{st}^{-1}\left(B_{\omega}\right)\right)$. Hence, for $L(P)$-almost all $\omega$, there exists $n$ infinite so that $\nu_{\omega}^{n}$ is not standardly distributed.

\section{REFERENCES}

1. Robert M. Anderson, A non-standard representation for Brownian motion and Ito integration, Israel $\mathbf{J}$. Math. 25 (1976), 15-46.

2. Star-finite probability theory, Ph.D. Dissertation, Yale Univ., New Haven, Conn., 1977.

3. Strong core theorems with nonconvex preferences, Cowles Foundation Discussion Paper No. 590, Yale Univ., New Haven, Conn., 1981.

4. Robert M. Anderson and Salim Rashid, A nonstandard characterization of weak convergence, Proc. Amer. Math. Soc. 69 (1978), 327-332.

5. Allen R. Bernstein and Frank Wattenberg, Nonstandard measure theory, Applications of Model Theory to Algebra. Analysis, and Probability (W. A. J. Luxemburg, editor), Holt, Rinehart and Winston. New York, 1969, pp. 171-185.

6. Patrick Billingsley, Convergence of probability measures, Wiley, New York, 1968.

7. Nelson Dunford and Jacob T. Schwartz, Linear operators. Part I, Wiley, New York, 1957.

8. Lester L. Helms, Mean convergence of martingales, Trans. Amer. Math. Soc. 87 (1958), 439-446.

9. Ward Henson, On the nonstandard representation of measures, Trans. Amer. Math. Soc. 172 (1972), 437-446.

10. Douglas N. Hoover, Probability logic, Ann. Math. Logic 14 (1978), 287-313.

11. H. Jerome Keisler, Hyperfinite model theory, Logic Colloquium 76 (R. O. Gandy and J. M. E. Hyland, editors), North-Holland, Amsterdam, 1977, pp. 5-110.

12. Peter A. Loeb, A nonstandard representation of measurable spaces and $L^{\infty}$, Bull. Amer. Math. Soc. 77 (1971), 540-544.

13. A nonstandard representation of measurable spaces, $L_{\infty}$, and $L_{\infty}^{*}$, Contributions to Non-standard Analysis (W. A. J. Luxemburg and A. Robinson, editors), North-Holland, Amsterdam, 1972, pp. 65-80.

14. A nonstandard representation of Borel measures and $\sigma$-finite measures, Victoria Symposium on Nonstandard Analysis (A. Hurd and P. Loeb, editors), Lecture Notes in Math., vol. 369, SpringerVerlag, Berlin and New York, 1974.

15. Conversion from nonstandard to standard measure spaces and applications in probability theory, Trans. Amer. Math. Soc. 211 (1975), 113-122.

16. A _ Applications of nonstandard analysis to ideal boundaries in potential theory, Israel J. Math. 25 (1976), 154-187.

17. W. A. J. Luxemburg, A general theory of monads, Applications of Model Theory to Algebra, Analysis, and Probability (W. A. J. Luxemburg, editor), Holt, Rinehart and Winston, New York, 1969, pp. 18-86.

18. Moshe Machover and Joram Hirschfeld, Lectures on nonstandard analysis, Lecture Notes in Math., vol. 94, Springer-Verlag, Berlin and New York, 1969.

19. Rohit Parikh and Milton Parnes, Conditional probability can be defined for all pairs of sests of reals, Advances in Math. 9 (1972), 313-315.

20. Conditional probabilities and uniform sets, Victoria Symposium on Nonstandard Analysis (A. Hurd and P. Loeb, editors), Lecture Notes in Math., vol. 369, Springer-Verlag, Berlin and New York, 1974. 
21. Salim Rashid, Economies with infinitely many traders, Ph.D. Dissertation, Yale Univ., New Haven, Ct., 1976

22. Abraham Robinson, Non-standard analysis, North-Holland, Amsterdam, 1966.

23. Walter Rudin, Real and complex analysis, McGraw-Hill, New York, 1974.

24. K. D. Stroyan and W. A. J. Luxemburg, Introduction to the theory of infinitesimals, Academic Press, New York, 1976.

25. Douglas N. Hoover and Edwin Perkins, Nonstandard construction of the stochastic integral and applications to stochastic differential equations. I, II (preprint).

26. Tom L. Lindstrom, Hyperfinite stochastic integration. I, II, III, and Addendum, Math. Scand. 46 (1980), 265-333.

27. Peter A. Loeb, Weak limits of measures and the standard part map, Proc. Amer. Math. Soc. 77 (1979), 128-135.

28. __ An introduction to nonstandard analysis and hyperfinite probability theory, Probabilistic Analysis and Related Topics, vol. 2 (A. T. Bharucha-Reid, editor), Academic Press, New York, 1979, pp. 105-142.

29. Frank Wattenberg, Nonstandard measure theory: Avoiding pathological sets, Trans. Amer. Math. Soc. 250 (1979), 357-368.

Department of Mathematics and Economics, Princeton University, Princeton, New Jersey 08544 